Tim Kobert · Claus Scheiderer(1)

\title{
Spectrahedral representation of polar orbitopes
}

Received: 10 January 2021 / Accepted: 4 August 2021 / Published online: 18 August 2021

\begin{abstract}
Let $K$ be a compact Lie group and $V$ a finite-dimensional representation of $K$. The orbitope of a vector $x \in V$ is the convex hull $\mathcal{O}_{x}$ of the orbit $K x$ in $V$. We show that if $V$ is polar then $\mathcal{O}_{x}$ is a spectrahedron, and we produce an explicit linear matrix inequality representation. We also consider the coorbitope $\mathcal{O}_{x}^{o}$, which is the convex set polar to $\mathcal{O}_{x}$. We prove that $\mathcal{O}_{x}^{o}$ is the convex hull of finitely many $K$-orbits, and we identify the cases in which $\mathcal{O}_{x}^{o}$ is itself an orbitope. In these cases one has $\mathcal{O}_{x}^{o}=c \cdot \mathcal{O}_{x}$ with $c>0$. Moreover we show that if $x$ has "rational coefficients" then $\mathcal{O}_{x}^{o}$ is again a spectrahedron. This provides many new families of doubly spectrahedral orbitopes. All polar orbitopes that are derived from classical semisimple Lie algebras can be described in terms of conditions on singular values and Ky Fan matrix norms.
\end{abstract}

\section{Introduction}

Let $K$ be a compact Lie group, and let $V$ be a finite-dimensional real representation of $K$. The orbitope of a vector $x \in V$, denoted $\mathcal{O}_{x}$, is the convex hull of the orbit $K x$ in $V$. Orbitopes are highly symmetric objects that are interesting from many perspectives, like convex geometry, algebraic geometry, Lie theory, symplectic geometry, combinatorial geometry or optimization. We refer to [18] for a broad overview with plenty of explicit examples.

Here our focus will be on properties of orbitopes that are particularly relevant to optimization, and more specifically, to semidefinite programming. We are interested in existence and explicit construction of spectrahedral representations for orbitopes and related convex bodies. For this we consider a particular class of group representations, namely polar representations of connected compact Lie groups. As far as the orbit structure is concerned, all such representations arise from Riemannian symmetric spaces $M=G / K$ as actions of the isotropy group on the tangent space at a point. In other words, each polar representation comes from a Cartan decomposition $\mathfrak{g}=\mathfrak{k} \oplus \mathfrak{p}$ of a real semisimple Lie algebra $\mathfrak{g}$, as the adjoint representation of $K$ on $\mathfrak{p}$. Dadok [7] showed that these representations have

T. Kobert · C. Scheiderer ( $\varangle$ ): Fachbereich Mathematik und Statistik, Universität Konstanz, 78457 Konstanz, Germany.

e-mail: claus.scheiderer@uni-konstanz.de

T. Kobert: e-mail: tim.kobert@uni-konstanz.de

Mathematics Subject Classification: 22E46 · 17B10 - 20G20 · 90C22

https://doi.org/10.1007/s00229-021-01337-z 
very particular properties. At the same time they comprise all the familiar actions of the classical unitary, orthogonal or symplectic groups on (skew-) hermitian or symmetric matrices.

Our main results are as follows. We prove that every orbitope $\mathcal{O}_{x}$ in a polar representation is a spectrahedron, i.e. an affine-linear slice of the psd matrix cone. In fact we produce an explicit linear matrix inequality representation for any such orbitope (Theorem 4.4). So far, this result was known only for a few scattered classes of examples. We also consider the dual convex body $\mathcal{O}_{x}^{o}$, called the coorbitope of $x$. We prove that $\mathcal{O}_{x}^{o}$ always is the convex hull of finitely many $K$-orbits, and we identify those orbits explicitly (Corollary 6.6). In particular, we isolate the cases when $\mathcal{O}_{x}$ is a biorbitope (Theorem 6.7), meaning that $\mathcal{O}_{x}^{o}$ is an orbitope as well. Remarkably, $\mathcal{O}_{x}$ is always self-polar up to positive scaling when it is a biorbitope (Theorem 6.9). Moreover, we show that whenever the orbitope $\mathcal{O}_{x}$ can be "defined over the rational numbers $\mathbb{Q}$ ", the coorbitope $\mathcal{O}_{x}^{o}$ is again a spectrahedron, and we find an explicit linear matrix inequality for it (Theorem 7.2). So far, only very few examples of doubly spectrahedral sets were known [20]. Our result provides many new series of sets with this property.

The main and all-important tool for our results is Kostant's convexity theorem [13]. It allows to reduce most questions considered here to a Cartan subspace, and even to a Weyl chamber. In this way the questions become polyhedral in nature.

The paper is organized as follows. Polar representations and orbitopes are recalled in Sect. 1. The general background on semisimple real Lie algebras and their restricted root systems is summarized in Sect. 2, as far as it will be needed here. Kostant's theorem is stated in Sect. 3, together with a few immediate consequences. Spectrahedral representations for polar orbitopes are constructed in Sect. 4. In Sects. 5 and 6 we relate the facial structure of $\mathcal{O}_{x}$ to the momentum polytope $P_{x}$, with an emphasis on maximal faces of $\mathcal{O}_{x}$ resp. facets of $P_{x}$. Since the maximal faces of $\mathcal{O}_{x}$ correspond to the extreme points of the polar set $\mathcal{O}_{x}^{o}$, this allows us to identify biorbitopes. Doubly spectrahedral orbitopes are considered in Sect. 7. Finally, in Sect. 8 we list all polar orbitopes that are derived from semisimple Lie algebras of classical type. All these orbitopes have descriptions in terms of singular values of matrices over $\mathbb{R}, \mathbb{C}$ or $\mathbb{H}$. Typically, they consist of intersections of balls of various radii with respect to different Ky Fan matrix norms.

Some of the results presented here are taken from the 2018 doctoral thesis [12] of the first author, written under the guidance of the second.

\section{Polar representations and orbitopes}

1.1. We recall the notion of polar representation, following Dadok [7]. Let $K$ be a Lie group with Lie algebra $\mathfrak{k}$, and let $K \rightarrow O(V)$ be a linear representation of $K$ on a (finite-dimensional) real vector space $V$, preserving a fixed inner product. For every $x \in V$, the linear subspace $\mathfrak{a}_{x}=(\mathfrak{k} x)^{\perp}$ of $V$ meets every $K$-orbit [7, Lemma 1]. A vector $x \in V$ is said to be regular if the orbit $K x$ has maximal dimension. The subspaces $\mathfrak{a}_{x}$, for $x$ regular, are called the Cartan subspaces of the representation. The Cartan subspaces are all $K$-conjugate if, and only if, they are 
orthogonal to the $K$-orbits passing through them [7, Prop. 2]. The representation $\rho$ is said to be polar if these equivalent conditions are satisfied.

Every Riemannian symmetric space $M$ gives rise to a polar representation, namely the action of the isotropy group on the tangent space $T_{e}(M)$ at a point $e \in M$. In other words, let $G$ be a connected real semisimple Lie group with Lie algebra $\mathfrak{g}$, let $\mathfrak{g}=\mathfrak{k} \oplus \mathfrak{p}$ be a Cartan decomposition, and let $K \subseteq G$ be the analytic subgroup corresponding to k. Then $K$ is a maximal compact subgroup of $G$, and the adjoint action of $K$ on $\mathfrak{p}$ is an example of a polar representation. This representation is irreducible if and only if $\mathfrak{g}$ is simple as a Lie algebra. Conversely, as far as the orbit structure is concerned, these are the only examples of polar representations of connected Lie groups:

Proposition 1.2. (Dadok [7, Proposition 6]) Let $V$ be a polar representation of a connected Lie group $H$. There is a real semisimple Lie algebra $\mathfrak{g}$ with Cartan decomposition $\mathfrak{g}=\mathfrak{k} \oplus \mathfrak{p}$, together with a vector space isomorphism $f: V \rightarrow \mathfrak{p}$, such that $f(H \cdot x)=\operatorname{Ad}(K) \cdot f(x)$ for every $x \in V$, where $K \subseteq \operatorname{Aut}(\mathfrak{g})$ is the analytic subgroup with Lie algebra $\mathfrak{k}$.

Remark 1.3. Let $V=\bigoplus_{i=1}^{n} V_{i}$ be the irreducible decomposition of an arbitrary polar representation of $K$. Then each irreducible summand $V_{i}$ is again a polar representation of $K$ (Dadok [7, Theorem 4(i)]). Moreover, if $x=\sum_{i=1}^{n} x_{i} \in V$ with $x_{i} \in V_{i}$ for all $i$, then $\mathcal{O}_{x}=\mathcal{O}_{x_{1}} \times \cdots \times \mathcal{O}_{x_{n}}$ with $\mathcal{O}_{x_{i}}=\operatorname{conv}_{V_{i}}\left(K x_{i}\right)$. Indeed, such a direct product decomposition holds for the $K$-orbit of $x$ by Dadok's theorem, and hence it holds for the convex hulls as well. In other words, every polar orbitope is a cartesian direct product of irreducible polar orbitopes.

1.4. Recall that a spectrahedron in $\mathbb{R}^{n}$ is the solution set of a linear matrix inequality (LMI). So $S \subseteq \mathbb{R}^{n}$ is a spectrahedron if there exist complex hermitian matrices $A_{0}, \ldots, A_{n}$ of some size $d \times d$ such that

$$
S=\left\{x \in \mathbb{R}^{n}: A_{0}+\sum_{i=1}^{n} x_{i} A_{i} \succeq 0\right\},
$$

where $A \succeq 0$ means that $A$ is positive semidefinite (all eigenvalues are nonnegative). Note that an LMI with complex hermitian $d \times d$ matrices may be converted into an equivalent LMI with real symmetric $2 d \times 2 d$ matrices, which is why spectrahedra are often defined via real symmetric LMIs.

1.5. Let $V$ be a linear representation (real and finite-dimensional) of a compact Lie group $K$. Given $x \in V$, the convex hull $\operatorname{conv}(K x)$ of the orbit of $x$ in $V$ is called the $(K-)$ orbitope of $x$. We usually denote it by $\mathcal{O}_{x}=\operatorname{conv}(K x)$, assuming that $K$ and $V$ are understood. The orbitope $\mathcal{O}_{x}$ is a compact convex set on which $K$ acts, and whose set of extreme points coincide with the orbit $K x$.

We will study orbitopes $\mathcal{O}_{x}=\operatorname{conv}(K x)$ in polar representations $V$ of compact connected Lie groups $K$. Using Proposition 1.2, we can and will always assume that $V=\mathfrak{p}$ where $\mathfrak{g}=\mathfrak{k} \oplus \mathfrak{p}$ is a Cartan decomposition of a real semisimple Lie algebra $\mathfrak{g}$, and that the action is the adjoint action of the analytic subgroup $K$ of $\operatorname{Aut}(\mathfrak{g})$. 
1.6. If $V$ is a vector space over $\mathbb{R}$, the dual vector space is denoted by $V^{\vee}=$ $\operatorname{Hom}(V, \mathbb{R})$. The convex hull of a set $M \subseteq V$ is written $\operatorname{conv}(M)$. Our notation for matrix groups and matrix Lie algebras tries to follow the conventions in [11]. In particular, $S U(n), S O(n), S p(n)$ are the classical compact Lie groups, $s u(n)$, $s o(n), \operatorname{sp}(n)$ are their Lie algebras, etc. The diagonal $n \times n$ matrix with diagonal entries $a_{1}, \ldots, a_{n}$ is denoted $\operatorname{diag}\left(a_{1}, \ldots, a_{n}\right)$.

\section{Background on semisimple real Lie algebras}

We use standard notation and terminology for semisimple Lie groups and Lie algebras, and we'll recall it briefly here. As a general reference we refer to Knapp's monograph [11], in particular to Chapter 6.

2.1. Let $\mathfrak{g}$ be a semisimple Lie algebra over $\mathbb{R}$, hence a finite direct sum of simple (nonabelian) Lie algebras over $\mathbb{R}$. Recall that if $\mathfrak{g}$ is simple then either $\mathfrak{g}$ has a structure as a (simple) Lie algebra over $\mathbb{C}$, or else $\mathfrak{g} \mathbb{C}:=\mathfrak{g} \otimes \mathbb{C}$ is a simple Lie algebra over $\mathbb{C}$.

Let $\theta$ be a Cartan involution on $\mathfrak{g}$ and $\mathfrak{g}=\mathfrak{k} \oplus \mathfrak{p}$ the corresponding Cartan decomposition. With respect to the Killing form $\langle-,-\rangle$ of $\mathfrak{g}$, the decomposition $\mathfrak{k} \oplus \mathfrak{p}$ is orthogonal, and the restriction of $\langle-,-\rangle$ to $\mathfrak{k}$ (resp. $\mathfrak{p}$ ) is negative (resp. positive) definite.

2.2. Choose a maximal commutative subspace $\mathfrak{a}$ of $\mathfrak{p}$, and let $\Sigma \subseteq \mathfrak{a}^{\vee}=\operatorname{Hom}(\mathfrak{a}, \mathbb{R})$ be the system of restricted roots of $(\mathfrak{g}, \mathfrak{a})$. This is an abstract root system, possibly non-reduced. Fixing an ordering on $\mathfrak{a}^{\vee}$ we have the sets $\Sigma_{+} \subseteq \Sigma$ of positive restricted roots and $\Gamma=\left\{\beta_{1}, \ldots, \beta_{n}\right\} \subseteq \Sigma_{+}$of simple restricted roots.

2.3. Let $\mathfrak{t}$ be a maximal commutative subalgebra of the centralizer $Z_{\mathfrak{k}}(\mathfrak{a})$ of $\mathfrak{a}$ in $\mathfrak{k}$. Then $\mathfrak{h}:=i \mathfrak{t} \oplus \mathfrak{a}$ is a commutative subalgebra of $\mathfrak{g}_{\mathbb{C}}$ (the complexification of $\mathfrak{g}$ ) for which $\mathfrak{h}_{\mathbb{C}}$ is a Cartan algebra of $\mathfrak{g}_{\mathbb{C}}$. The rank of $\mathfrak{g}_{\mathbb{C}}$ will be denoted by $l=\operatorname{dim}(\mathfrak{h})$, the real rank of $\mathfrak{g}$ by $n=\operatorname{dim}(\mathfrak{a})$.

The Killing form of $\mathfrak{g}_{\mathbb{C}}$ restricted to $\mathfrak{h}$ is a euclidean inner product on $\mathfrak{h}$, denoted by $\langle-,-\rangle$. All roots of $\left(\mathfrak{g}_{\mathbb{C}}, \mathfrak{h}_{\mathbb{C}}\right)$ are real-valued on $\mathfrak{h}$. We let $\Delta \subseteq \mathfrak{h}^{\vee}=\operatorname{Hom}(\mathfrak{h}, \mathbb{R})$ be the root system of $\left(\mathfrak{g}_{\mathbb{C}}, \mathfrak{h}_{\mathbb{C}}\right)$. Extending the ordering on $\mathfrak{a}^{\vee}$ suitably to $\mathfrak{h}^{\vee}$ (e.g. take the lexicographic order on $\mathfrak{h}^{\vee}=(\mathfrak{a}+i \mathfrak{t})^{\vee}$ with $\mathfrak{a}$ before $i \mathfrak{t}[11$, p. 377]), we let $\Delta_{+} \subseteq \Delta$ be the positive roots and $\Pi=\left\{\alpha_{1}, \ldots, \alpha_{l}\right\} \subseteq \Delta_{+}$the simple roots.

2.4. Let $G=\operatorname{Aut}(\mathfrak{g})_{0}$, the identity component of the automorphism group of $\mathfrak{g}$. The Lie algebra of $G$ is naturally identified with $\mathfrak{g}$. The analytic subgroup $K \subseteq G$ with Lie algebra $\mathfrak{k}$ is a maximal compact subgroup of $G$, and $K$ acts on (k and) $\mathfrak{p}$ via the adjoint action. We denote this action simply by $g x:=\operatorname{Ad}(g) x$, for $g \in K$ and $x \in \mathfrak{p}$. Every element of $\mathfrak{p}$ is $K$-conjugate to an element of $\mathfrak{a}$. Note that the $K$-action preserves the quadratic (Killing) form on $\mathfrak{p}$. This action is a polar representation of $K$, and as far as the orbit structure is concerned, every polar representation of a connected Lie group arises in this way (Proposition 1.2). 
2.5. Note that the direct sum decomposition $\mathfrak{h}=i \mathfrak{t} \oplus \mathfrak{a}$ is orthogonal with respect to the Killing form. The restriction map $r: \mathfrak{h}^{\vee} \rightarrow \mathfrak{a}^{\vee}$ satisfies $r\left(\Delta_{+}\right) \subseteq \Sigma_{+} \cup\{0\}$ and $r(\Pi) \subseteq \Gamma \cup\{0\}$. Conversely, there exists an involution $\alpha \mapsto \alpha^{\prime}$ on $\Pi$ such that for every $\beta \in \Gamma$, the set $\{\alpha \in \Pi: r(\alpha)=\beta\}$ has the form $\left\{\alpha, \alpha^{\prime}\right\}$ with $\alpha \in \Pi$.

2.6. From the inner product $\langle-,-\rangle$ on $\mathfrak{h}$ we get linear isomorphisms $\mathfrak{h} \stackrel{\sim}{\rightarrow} \mathfrak{h}^{\vee}$ and $\mathfrak{a} \stackrel{\sim}{\rightarrow} \mathfrak{a}^{\vee}$. We use them to transfer the inner product from $\mathfrak{h}$ to $\mathfrak{h}^{\vee}$ and from $\mathfrak{a}$ to $\mathfrak{a}^{\vee}$.

For every restricted root $\beta \in \Sigma$ let $s_{\beta}: \mathfrak{a}^{\vee} \rightarrow \mathfrak{a}^{\vee}$ denote the root reflection $\gamma \mapsto \gamma-\frac{2\langle\beta, \gamma\rangle}{|\beta|^{2}} \beta$. We always write $W=\left\langle s_{\beta}: \beta \in \Sigma\right\rangle$ for the (restricted) Weyl group of $(\mathfrak{g}, \mathfrak{a})$. Via the identification $\mathfrak{a} \stackrel{\sim}{\rightarrow} \mathfrak{a}^{\vee}$ we consider $W$ as a reflection group on $\mathfrak{a}$ as well: For $\beta \in \Sigma$, if $h_{\beta} \in \mathfrak{a}$ is the element with $\left\langle h_{\beta}, x\right\rangle=\beta(x)$ for all $x \in \mathfrak{a}$, then $s_{\beta}$ acts on $\mathfrak{a}$ by $s_{\beta}(x)=x-\frac{2 \beta(x)}{\left|h_{\beta}\right|^{2}} h_{\beta}$. It is well known that $W$ is naturally isomorphic to $N_{K}(\mathfrak{a}) / Z_{K}(\mathfrak{a})[11,6.57]$.

We always denote by

$$
C=\left\{x \in \mathfrak{a}: \beta_{1}(x) \geq 0, \ldots, \beta_{n}(x) \geq 0\right\}
$$

the (closed) Weyl chamber for the action of $W$. So $C$ is a polyhedral convex cone, and every element of $\mathfrak{a}$ is $W$-conjugate to a unique element of $C$.

2.7. Let $\lambda_{1}, \ldots, \lambda_{l}$ be the basis of $\mathfrak{h}^{\vee}$ that is dual to $\Pi=\left\{\alpha_{1}, \ldots, \alpha_{l}\right\}$, so $\left\langle\alpha_{i}, \lambda_{k}\right\rangle=$ $\delta_{i k}$ for $i, k=1, \ldots, l$. Similarly, let $\mu_{1}, \ldots, \mu_{n} \in \mathfrak{a}^{\vee}$ be defined by $\left\langle\beta_{j}, \mu_{k}\right\rangle=\delta_{j k}$ for $j, k=1, \ldots, n$. The fundamental weights of $\left(\mathfrak{g}_{\mathbb{C}}, \mathfrak{h}_{\mathbb{C}}\right)$ are the linear forms $\omega_{i} \in$ $\mathfrak{h}^{\vee}$ defined by $\omega_{i}=\frac{1}{2}\left|\alpha_{i}\right|^{2} \lambda_{i}(i=1, \ldots, l)$, so $\frac{2\left\langle\omega_{i}, \alpha_{k}\right\rangle}{\left|\alpha_{k}\right|^{2}}=\delta_{i k}$ for $i, k=1, \ldots, l$. For each linear combination $\omega=\sum_{i=1}^{l} m_{i} \omega_{i}$ with integer coefficients $m_{i} \geq 0$, there exists a unique (up to isomorphism) irreducible representation of $\mathfrak{g}_{\mathbb{C}}$ with highest weight $\omega$. The irreducible representation of $\mathfrak{g}_{\mathbb{C}}$ with highest weight $\omega_{i}$ is called the $i$ th fundamental representation of $\mathfrak{g}_{\mathbb{C}}$, we'll denote it by $\rho_{i}(i=1, \ldots, l)$.

2.8. As before, let $\Pi \subseteq \Delta_{+} \subseteq \mathfrak{h}^{\vee}$ resp. $\Gamma \subseteq \Sigma_{+} \subseteq \mathfrak{a}^{\vee}$ be the systems of simple resp. simple restricted roots. We need to relate the dual bases of $\Pi$ and $\Gamma$ to each other. Given $\alpha \in \Pi$, let $\lambda_{\alpha} \in \mathfrak{h}^{\vee}$ be defined by $\left\langle\lambda_{\alpha}, \alpha^{\prime}\right\rangle=\delta_{\alpha, \alpha^{\prime}}$ for each $\alpha^{\prime} \in \Pi$. Given $\beta \in \Gamma$, let $\mu_{\beta} \in \mathfrak{a}^{\vee}$ be defined by $\left\langle\mu_{\beta}, \beta^{\prime}\right\rangle=\delta_{\beta, \beta^{\prime}}$ for each $\beta^{\prime} \in \Gamma$. (So if $\alpha=\alpha_{i}$ then $\lambda_{\alpha}=\lambda_{i}$, and similarly if $\beta=\beta_{j}$ then $\mu_{\beta}=\mu_{j}$.) The following fact is certainly well-known, but we haven't been able to find a suitable reference for it:

Lemma 2.9. Let $\alpha \in \Pi$. If $\beta:=r(\alpha) \neq 0$ then $r\left(\lambda_{\alpha}\right)=q \mu_{\beta}$ for some rational number $q>0$. If $r(\alpha)=0$ then $r\left(\lambda_{\alpha}\right)=0$.

In fact the argument shows that $r\left(\lambda_{\alpha}\right)=\mu_{\beta}$ if $\alpha$ is the only element of $\Pi$ that restricts to $\beta$, and $r\left(\lambda_{\alpha}\right)=\frac{1}{2} \mu_{\beta}$ if there are two such elements.

Proof. Let $\mathfrak{a}^{\vee} \rightarrow \mathfrak{h}^{\vee}, \mu \mapsto \tilde{\mu}$ denote the linear map that is adjoint to the restriction $\operatorname{map} r: \mathfrak{h}^{\vee} \rightarrow \mathfrak{a}^{\vee}$, so $\langle\lambda, \widetilde{\mu}\rangle=\langle r(\lambda), \mu\rangle$ for $\lambda \in \mathfrak{h}^{\vee}$ and $\mu \in \mathfrak{a}^{\vee}$. Let $\Pi_{0}=\{\alpha \in \Pi$ : $r(\alpha)=0\}$ and $\Pi_{1}=\Pi \backslash \Pi_{0}$. The Cartan involution $\theta$ of $\mathfrak{g}$ induces id on $i \mathfrak{t}$ and -id on $\mathfrak{a}$. The dual involution $\theta^{\vee}$ on $\mathfrak{h}^{\vee}$ satisfies $-\theta^{\vee}(\Pi) \subseteq \Pi$. For $\alpha \in \Pi$ we 
abbreviate $\alpha^{\prime}:=-\theta^{\vee}(\alpha)$. If $\alpha \in \Pi_{1}$, then the only elements of $\Pi$ that restrict to $\beta=r(\alpha)$ are $\alpha$ and $\alpha^{\prime}$ (see [4, Prop. 29.9] for these facts).

Let $\beta \in \Gamma$ be a restricted simple root, and let $\alpha \in \Pi$ with $r(\alpha)=\beta$. From $\alpha^{\prime}=-\theta^{\vee}(\alpha)$ we see $\alpha+\alpha^{\prime}=2 \widetilde{\beta}$. If $\lambda \in \mathfrak{h}^{\vee}$ is dominant, i.e. satisfies $\langle\lambda, \gamma\rangle \geq 0$ for all $\gamma \in \Pi$, we conclude $\langle r(\lambda), \beta\rangle=\langle\lambda, \widetilde{\beta}\rangle=\frac{1}{2}\left\langle\lambda, \alpha+\alpha^{\prime}\right\rangle \geq 0$. This shows

$$
\operatorname{cone}\left(r\left(\lambda_{1}\right), \ldots, r\left(\lambda_{l}\right)\right) \subseteq \operatorname{cone}\left(\mu_{1}, \ldots, \mu_{n}\right)
$$

in $\mathfrak{a}^{\vee}$.

On the other hand, for $\gamma \in \Pi$ the inner product $\left\langle\widetilde{\mu_{\beta}}, \gamma\right\rangle=\left\langle\mu_{\beta}, r(\gamma)\right\rangle$ is 1 if $r(\gamma)=\beta$, and 0 otherwise. Therefore $\widetilde{\mu_{\beta}}=\frac{1}{2}\left(\lambda_{\alpha}+\lambda_{\alpha^{\prime}}\right)$. In particular, $\mu_{\beta}=r\left(\tilde{\mu}_{\beta}\right)=\frac{1}{2} r\left(\lambda_{\alpha}+\lambda_{\alpha^{\prime}}\right)$, which proves the reverse inclusion of (1).

If $\alpha^{\prime}=\alpha$ then $r\left(\lambda_{\alpha}\right)=\mu_{\beta}$. It remains to consider the case $\alpha^{\prime} \neq \alpha$. From $\alpha+\alpha^{\prime}=2 \widetilde{\beta}$ we see $\left\langle r\left(\lambda_{\alpha}\right), \beta\right\rangle=\left\langle\lambda_{\alpha}, \widetilde{\beta}\right\rangle=\frac{1}{2}$, and similarly $\left\langle r\left(\lambda_{\alpha^{\prime}}\right), \beta\right\rangle=\frac{1}{2}$. On the other hand, $r\left(\lambda_{\alpha}+\lambda_{\alpha^{\prime}}\right)=2 \mu_{\beta}$, together with (1), implies that $r\left(\lambda_{\alpha}\right)$, $r\left(\lambda_{\alpha^{\prime}}\right)$ are positive scalar multiples of $\mu_{\beta}$, since $\mu_{\beta}$ generates an extreme ray of cone $\left(\mu_{1}, \ldots, \mu_{m}\right)$. Altogether it follows that $r\left(\lambda_{\alpha}\right)=r\left(\lambda_{\alpha^{\prime}}\right)=\frac{1}{2} \mu_{\beta}$.

Finally assume $r(\alpha)=0$, and let $\gamma \in \Pi$ be arbitrary with $\beta=r(\gamma) \neq 0$. Then $\left\langle r\left(\lambda_{\alpha}\right), \beta\right\rangle=\left\langle\lambda_{\alpha}, \widetilde{\beta}\right\rangle=\frac{1}{2}\left\langle\lambda_{\alpha}, \gamma+\gamma^{\prime}\right\rangle$ since $2 \widetilde{\beta}=\gamma+\gamma^{\prime}$, see above. But $\alpha \notin\left\{\gamma, \gamma^{\prime}\right\}$ since $r(\gamma)=r\left(\gamma^{\prime}\right)=\beta \neq 0$. Hence $\left\langle r\left(\lambda_{\alpha}\right), \beta\right\rangle=0$. This for all $\beta \in \Gamma$ shows $r\left(\lambda_{\alpha}\right)=0$.

\section{Kostant's convexity theorem}

We assume the setup of Sect. 2. So $\mathfrak{g}$ is a semisimple real Lie algebra with Cartan decomposition $\mathfrak{g}=\mathfrak{k} \oplus \mathfrak{p}$ and maximal abelian subspace $\mathfrak{a}$ of $\mathfrak{p}$. This gives us the system $\Sigma \subseteq \mathfrak{a}^{\vee}$ of reduced roots of $(\mathfrak{g}, \mathfrak{a})$, on which the reduced Weyl group $W$ acts. After fixing an ordering we have the simple positive roots $\Gamma$ and the Weyl chamber $C \subseteq \mathfrak{a}$.

The key technical tool for this paper is Kostant's convexity theorem, together with its consequences. To a large extent, it allows to reduce the study of the polar orbits $K x$ and their orbitopes $\mathcal{O}_{x}$ to a Weyl chamber, whereby the $K$-action on $\mathfrak{p}$ gets replaced by the $W$-action on $\mathfrak{a}$. We now recall this theorem.

3.1. Let $T \subseteq \mathfrak{a}$ be the cone that is dual to $C$ (with respect to the $W$-invariant inner product). If $\Gamma=\left\{\beta_{1}, \ldots, \beta_{n}\right\}$ then $T=\left\{x \in \mathfrak{a}: \mu_{1}(x) \geq 0, \ldots, \mu_{n}(x) \geq 0\right\}$ where $\mu_{1}, \ldots, \mu_{n} \in \mathfrak{a}^{\vee}$ is the dual basis of $\Gamma$ as in 2.7. For $x \in \mathfrak{a}$, the convex hull $P_{x}:=\operatorname{conv}(W x)$ of the (restricted) Weyl group orbit of $x$ will play a central role. In Hamiltonian geometry, $P_{x}$ is called the momentum polytope associated to $x$ [17], a term that we will adopt. According to Kostant, $P_{x}$ is characterized as follows:

Proposition 3.2. [13, Lemma 3.3] Let $x \in C$ and $y \in \mathfrak{a}$.

(a) $y \in P_{x}$ if and only if $x-w y \in T$ for every $w \in W$.

(b) If $y \in C$ then $y \in P_{x}$ if and only if $x-y \in T$.

In particular, $P_{x} \cap C=\left\{y \in C: \mu_{j}(y) \leq \mu_{j}(x)\right.$ for $\left.j=1, \ldots, n\right\}$. 
Recall that the Killing form of $\mathfrak{g}$ restricts to a euclidean inner product on $\mathfrak{p}$. Let $\pi: \mathfrak{p} \rightarrow \mathfrak{a}$ denote the orthogonal projection from $\mathfrak{p}$ to $\mathfrak{a}$. Kostant's convexity theorem says:

Theorem 3.3. [13, Theorem 8.2] If $x \in \mathfrak{a}$ then $\pi(K x)=P_{x}$.

We record a few immediate consequences. Recall that $\mathcal{O}_{x}=\operatorname{conv}(K x)$ denotes the convex hull of the $K$-orbit of $x$ in $\mathfrak{p}$.

Corollary 3.4. If $x \in \mathfrak{a}$ then $\mathcal{O}_{x} \cap \mathfrak{a}=P_{x}$. Hence $\pi(\mathcal{O})=\mathcal{O} \cap \mathfrak{a}$ holds for every $K$-invariant convex subset $\mathcal{O}$ of/p.

Proof. Theorem 3.3 implies $\pi\left(\mathcal{O}_{x}\right)=P_{x}$, and hence $\mathcal{O}_{x} \cap \mathfrak{a} \subseteq P_{x}$. The reverse inclusion is obvious since $W x \subseteq K x$. The second assertion follows from the first, since every $K$-orbit in $\mathfrak{p}$ meets $\mathfrak{a}$.

Corollary 3.5. If $x, y \in \mathfrak{a}$ are $K$-conjugate then they are $W$-conjugate. Every $K$-orbit in $\mathfrak{p}$ intersects $C$ in a unique element.

Proof. Both statements are equivalent, so it suffices to prove the second. Let $x, y \in$ $C$ be $K$-conjugate. Then $P_{x}=P_{y}$ by Theorem 3.3, so Proposition 3.2(b) implies $\pm(x-y) \in T$, whence $x-y=0$.

Example 3.6. Let $n \geq 2$ and $\mathfrak{g}=\operatorname{sl}(n, \mathbb{R})$, the real $n \times n$ matrices of trace zero. The resulting polar representation is the natural action of the special orthogonal group $K=S O(n)$ on $\mathfrak{p}=\operatorname{sym}_{0}(n, \mathbb{R})$, the trace zero symmetric real matrices. The standard choice for $\mathfrak{a}$ is to take all diagonal matrices in $\mathfrak{p}$. The (restricted) Weyl group is $W=S_{n}$, the symmetric group, acting by permutation of the diagonal elements of $x \in \mathfrak{a}$. So the momentum polytope $P_{x}$ is the permutahedron of $x$, namely the convex hull of all permutations of $x$. Kostant's theorem 3.3 specializes to the (symmetric) Schur-Horn theorem (see [10, 4.3 .45 and 4.3.48], [14, 18]). Likewise, the hermitian version of the Schur-Horn theorem arises from Theorem 3.3 if we take $\mathfrak{k}=s u(n, \mathbb{C})$ and $\mathfrak{g}=\mathfrak{k}_{\mathbb{C}}$, so we get the adjoint action of the special unitary group $K=S U(n)$ on (traceless) hermitian $n \times n$ matrices, with the analogous theorem.

In Sect. 8 we will discuss examples of polar orbitopes in a systematic way.

\section{Polar orbitopes as spectrahedra}

4.1. In general, orbitopes under compact connected linear groups $K$ can't be expected to be spectrahedra. Examples are suitable $S O(2)$-orbitopes like the 4dimensional Barvinok-Novik orbitope [23], or the Grassmann orbitope $G_{3,6}$ of dimension 20 [18, Theorem 7.6], where the group $K=S O(6)$ is even semisimple. Using results from [21] it is easy to construct orbitopes under the 2-torus $K=S O(2) \times S O(2)$ which are not even linear projections of spectrahedra, for example the convex hull of $\left\{\left(s, s^{2}, s^{3}, t, t^{2}, s t, s t^{-1}\right): s, t \in \mathbb{C},|s|=|t|=1\right\}$ in $\mathbb{C}^{7}$. In fact it can be shown that in sufficiently high dimension, "most" (in a suitable sense) orbitopes under $S O(2) \times S O(2)$ fail to be projected spectrahedra [12]. 
Given this general situation, we think the following theorem all the more remarkable:

Theorem 4.2. Any orbitope in a polar representation $V$ of a connected Lie group $K$ is a spectrahedron.

4.3. By Proposition 1.2 we may assume that $\mathfrak{g}=\mathfrak{k} \oplus \mathfrak{p}$ is a Cartan decomposition of a real semisimple Lie algebra, and that $K$ has Lie algebra $\mathfrak{k}$ and acts on $V=\mathfrak{p}$ via the adjoint representation. We provide an explicit linear matrix inequality description for any such orbitope.

Fix $\mathfrak{a}, \mathfrak{h}$ together with compatible orderings (2.3), and let otherwise notation be as in Sect. 2. In particular, $\Pi=\left\{\alpha_{1}, \ldots, \alpha_{l}\right\}$ is the system of simple roots of $\left(\mathfrak{g}_{\mathbb{C}}, \mathfrak{h}_{\mathbb{C}}\right)$, and $\Gamma=\left\{\beta_{1}, \ldots, \beta_{n}\right\}$ is the system of simple restricted roots of $(\mathfrak{g}, \mathfrak{a})$. For each $j=1, \ldots, n$ choose an index $i=i(j) \in\{1, \ldots, l\}$ with $r\left(\alpha_{i(j)}\right)=\beta_{j}$, and let $\rho^{j}: \mathfrak{g}_{\mathbb{C}} \rightarrow \operatorname{End}\left(V_{j}\right)$ be the (complex) irreducible representation of $\mathfrak{g}_{\mathbb{C}}$ with highest weight $\omega_{i(j)}$. So $\rho^{j}$ is the $i(j)$ th fundamental representation of $\mathfrak{g}_{\mathbb{C}}$, see 2.7 . There exists an hermitian inner product on $V_{j}$ making $\rho^{j}(x)$ self-adjoint for all $x \in \mathfrak{p}$ (Lemma 4.6(a) below). In particular, $\rho^{j}(x)$ has real eigenvalues for every $x \in \mathfrak{p}$. A more precise version of Theorem 4.2 is:

Theorem 4.4. Given $x \in \mathfrak{p}$, the orbitope $\mathcal{O}_{x}=\operatorname{conv}(K x)$ consists of all $y \in \mathfrak{p}$ such that for each $j=1, \ldots, n$, all eigenvalues of $\rho^{j}(y)$ are less or equal than the largest eigenvalue of $\rho^{j}(x)$. In other words,

$$
\mathcal{O}_{x}=\left\{y \in \mathfrak{p}: \rho^{j}(y) \preceq c_{j} \cdot i d, j=1 \ldots, n\right\}
$$

where $c_{j}$ is the maximal eigenvalue of $\rho^{j}(x)$.

Upon choosing orthogonal bases of the representation spaces $V_{j}$, this is an explicit description of $\mathcal{O}_{x}$ by linear matrix inequalities, involving hermitian matrices in general. We remark that the fundamental representations $\rho^{j}$ of $\mathfrak{g}_{\mathbb{C}}$ are very well known and understood [24], in particular so for the classical Lie algebras.

Remark 4.5. Theorem 4.4 implies in particular that all faces in a polar orbitope are exposed, since this is true in every spectrahedron. This fact was proved before by Biliotti et al. [1]. Some spectrahedral representations contained in, or closely related to, Theorem 4.4 were constructed by Sanyal et al. [18], namely for symmetric Schur-Horn orbitopes (see Example 3.6), and also for skew-symmetric Schur-Horn orbitopes (see Example 8.7) and Fan orbitopes (see Example 4.8 and Remark 8.5). These latter orbitopes, as considered in [18], do not directly fall under the assumptions of Theorem 4.4, since the groups acting there are not connected (full instead of special orthogonal groups). It is not hard, however, to recover the results from [18] in our setup, see Remark 8.5. In Saunderson et al. [20], a spectrahedral representation for the convex hull of the special orthogonal group $S O(n)$ (and for its dual convex body) was found, see also Remark 4.9 and Example 7.7 below. Otherwise we believe that our result is new.

Before we give the proof of Theorem 4.4, recall the following well-known facts. 
Lemma 4.6. Let $\rho: \mathfrak{g}_{\mathbb{C}} \rightarrow \operatorname{End}(V)$ be a (complex) representation of $\mathfrak{g}_{\mathbb{C}}$.

(a) There exists an hermitian inner product on $V$ that makes $\rho(x)$ self-adjoint for every $x \in \mathfrak{p}$.

(b) If $\rho$ is irreducible with highest weight $\omega$, and if $x \in C$, then $\omega(x)$ is the largest eigenvalue of $\rho(x)$. (Recall that $C$ denotes the Weyl chamber, see 2.6.)

Proof. (a) Since $\mathfrak{g}_{1}:=\mathfrak{k} \oplus i \mathfrak{p} \subseteq \mathfrak{g}_{\mathbb{C}}$ is a compact real form of $\mathfrak{g}_{\mathbb{C}}$, there is an hermitian inner product on $V$ that is invariant under this Lie algebra, i.e. $\rho(y)$ is anti-self adjoint for every $y \in \mathfrak{g}_{1}$. In particular, $\rho(x)$ is self-adjoint for every $x \in \mathfrak{p}$.

(b) Let $\chi_{1}, \ldots, \chi_{r} \in \mathfrak{h}^{\vee}$ be the weights of $\rho$, with $\chi_{1}=\omega$. For $x \in \mathfrak{a}$, the eigenvalues of $\rho(x)$ are $\chi_{1}(x), \ldots, \chi_{r}(x)$. Every $\chi_{i}$ has the form $\omega-\sum_{i=1}^{l} k_{i} \alpha_{i}$ with integer coefficients $k_{i} \geq 0$. Since $x \in C$ we have $\alpha_{i}(x) \geq 0$ for each index $i$, from which the claim is obvious.

4.7. PROOF of Theorem 4.4. Let $x \in \mathfrak{p}$, let $c_{j}=\omega_{i(j)}(x)$ be the largest eigenvalue of $\rho^{j}(x)$, and write $O(x):=\left\{y \in \mathfrak{p}: \rho^{j}(y) \preceq c_{j} \cdot\right.$ id for $\left.j=1, \ldots, n\right\}$. Both sets $\mathcal{O}_{x}$ and $O(x)$ are $K$-invariant. To prove equality $\mathcal{O}_{x}=O(x)$, it therefore suffices to show $\mathcal{O}_{x} \cap C=O(x) \cap C$, since every $K$-orbit meets the Weyl chamber $C$ (Corollary 3.5).

So let $x, y \in C$. See 2.7 to Lemma 2.9 for notation in the following discussion. Since $\rho^{j}$ has highest weight $\omega_{i(j)}$, the largest eigenvalue of $\rho^{j}(y)$ is $\omega_{i(j)}(y)$ (Lemma 4.6(b)). Hence $y \in O(x)$ if and only if $\omega_{i(j)}(y) \leq \omega_{i(j)}(x)=c_{j}$ for $j=1, \ldots, n$. By Lemma 2.9, the restriction $r\left(\omega_{i(j)}\right) \in \mathfrak{a}^{\vee}$ is a positive scalar multiple of $\mu_{j}$ (recall that $\left.\omega_{i(j)}=\frac{1}{2}\left|\alpha_{i(j)}\right|^{2} \lambda_{i(j)}, j=1, \ldots, n\right)$. So $y \in O(x)$ if and only if $\mu_{j}(y) \leq \mu_{j}(x)$ for $j=1, \ldots, n$. By Proposition 3.2(b) this is equivalent to $y \in P_{x}$. On the other hand, $y \in P_{x}$ is equivalent to $y \in \mathcal{O}_{x}$ by Corollary 3.4.

Example 4.8. We illustrate the statement of Theorem 4.4. Let $n \geq 3$, and consider the action of $(g, h) \in K=S O(n) \times S O(n)$ on $x \in M_{n}(\mathbb{R})$ by $g x h^{t}$. This is a polar representation of $K$ that arises from the split real form of $D_{n}$, i.e. from the simple Lie algebra

$$
\mathfrak{g}=\operatorname{so}(n, n)=\left\{x \in M_{2 n}(\mathbb{R}): j x+x^{t} j=0\right\}, \quad j=\left(\begin{array}{cc}
I_{n} & 0 \\
0 & -I_{n}
\end{array}\right) .
$$

Note that $\mathfrak{g}$ consists of all block matrices

$$
x=\left(\begin{array}{cc}
u & w \\
w^{t} & v
\end{array}\right)
$$

with $u, v, w \in M_{n}(\mathbb{R})$ and $u, v$ skew-symmetric, and $\mathfrak{p} \subseteq \mathfrak{g}$ is the subspace of all symmetric such matrices, i.e. with $u=v=0$. As maximal commutative subspace of $\mathfrak{p}$ we take the space $\mathfrak{a}$ of all matrices (3) with $u=v=0$ and $w=$ $\operatorname{diag}\left(x_{1}, \ldots, x_{n}\right)$ diagonal. Denote such a matrix by $x=\left(x_{1}, \ldots, x_{n}\right)$. The simple roots $\beta_{i}=\alpha_{i}$ act on $x$ as $\alpha_{i}(x)=x_{i}-x_{i+1}(1 \leq i<n)$ and $\alpha_{n}(x)=x_{n-1}+x_{n}$. 
Hence the Weyl chamber $C$ consists of all $x \in \mathfrak{a}$ with $x_{1} \geq \cdots \geq x_{n-1} \geq\left|x_{n}\right|$. The fundamental weights $\mu_{i}=\lambda_{i}$ are $\lambda_{i}(x)=x_{1}+\cdots+x_{i}(i \leq n-2)$ and

$$
\lambda_{n-1}(x)=\frac{1}{2}\left(x_{1}+\cdots+x_{n-1}-x_{n}\right), \quad \lambda_{n}(x)=\frac{1}{2}\left(x_{1}+\cdots+x_{n-1}+x_{n}\right) .
$$

By Proposition 3.2, the momentum polytope for $x \in C$ is described by $P_{x} \cap C=$ $\left\{y \in C: \lambda_{i}(y) \leq \lambda_{i}(x), i=1, \ldots, n\right\}$. The first fundamental representation $\rho_{1}$ of $\mathfrak{g}$ is the natural representation (2), the higher ones are the exterior powers $\rho_{i}=\Lambda^{i} \rho_{1}$ $(1 \leq i \leq n-2)$. Moreover, $\rho_{n-1}$ and $\rho_{n}$ are the two half-spin representations. So $\operatorname{dim}\left(\rho_{i}\right)=\left(\begin{array}{c}2 n \\ i\end{array}\right)$ for $i \leq n-2$, and $\operatorname{dim}\left(\rho_{n-1}\right)=\operatorname{dim}\left(\rho_{n}\right)=2^{n-1}$. Expressing the $\rho_{i}$ by matrices one arrives at explicit spectrahedral representations of the $K$ orbitopes $\mathcal{O}_{x}$, for $x \in M_{n}(\mathbb{R})$. These representations are closely related to [18, Theorem 4.7], where the group acting is $O(n) \times O(n)$ instead of our $K$.

Remark 4.9. For general $x \in \mathfrak{p}$, none of the $n$ linear matrix inequalities describing $\mathcal{O}_{x}$ in Theorem 4.4 can be left out. For special $x$ this may be different. We illustrate this remark with just one example, deferring a detailed discussion to a later occasion.

Consider again the action of $K=S O(n) \times S O(n)$ on $M_{n}(\mathbb{R})$, as in Example 4.8, and take $x=I_{n} \in M_{n}(\mathbb{R})$, the identity matrix, so $x=(1, \ldots, 1)$ in notation of Example 4.8. The orbitope is $\mathcal{O}_{x}=\operatorname{conv} S O(n)$, the convex hull of the group $S O(n)$. Due to the special choice of $x$, the description of the momentum polytope simplifies. For $y \in C$, the condition $y_{1} \leq 1$ implies $\sum_{i=1}^{k} y_{i} \leq k$ for every $k=1, \ldots, n$. So $P_{x} \cap C$ is already described by the two inequalities $\lambda_{1}(y) \leq 1$ and $\lambda_{n-1}(y) \leq \frac{n-2}{2}$. We conclude that $\mathcal{O}_{x}=\operatorname{conv} S O(n)$ satisfies

$$
\operatorname{conv} S O(n)=\left\{y \in M_{n}(\mathbb{R}):\left(\begin{array}{cc}
0 & y \\
y^{t} & 0
\end{array}\right) \preceq I, \rho_{n-1}\left(\begin{array}{cc}
0 & y \\
y^{t} & 0
\end{array}\right) \preceq \frac{n-2}{2} I\right\}
$$

since both sets agree when intersected with $C$. This recovers one of the main results of Saunderson et al. [20, Theorem 1.3]. (In the notation of loc. cit., given a matrix $y=\left(y_{i j}\right) \in M_{n}(\mathbb{R})$, the $2^{n-1} \times 2^{n-1}$ matrix $\sum_{i, j=1}^{n} y_{i j} A^{(i j)}$ constructed there corresponds to the endomorphism $\rho_{n}\left(\begin{array}{cc}0 & 2 y \\ 2 y^{t} & 0\end{array}\right)$. The extra factor 2 accounts for the apparent difference between their result and ours.) See Example 7.7 below for a spectrahedral representation of the polar convex set $S O(n)^{o}$.

\section{Face correspondence}

5.1. As before let $\mathfrak{g}=\mathfrak{k} \oplus \mathfrak{p}$ be a Cartan decomposition of a semisimple real Lie algebra $\mathfrak{g}$. For general setup and notation see Sect. 2 . We continue to denote the orthogonal projection $\mathfrak{p} \rightarrow \mathfrak{a}$ by $\pi$. Let $x \in \mathfrak{p}$, and let $P_{x}$ be the momentum polytope of $x$ (3.1). If $Q$ is any face of $P_{x}$, then $F_{Q}:=\mathcal{O}_{x} \cap \pi^{-1}(Q)$ is a face of $\mathcal{O}_{x}$. For any $w \in W$ there exists $g \in N_{K}(\mathfrak{a})$ with $w=g Z_{K}(\mathfrak{a})$. The projection $\pi: \mathfrak{p} \rightarrow \mathfrak{a}$ is easily seen to commute with the action of $N_{K}(\mathfrak{a})$, and therefore $F_{w Q}=g F_{Q}$ holds. Hence the assignment $Q \mapsto F_{Q}$ induces a map from $W$-orbits of faces of $P_{x}$ to $K$-orbits of faces of $\mathcal{O}_{x}$. 
The following theorem asserts, in particular, that this map is bijective. This fact was originally proved by Biliotti et al. [1, Theorem 1.1]. We give a new proof that we think is considerably easier. Note however that [1] proves a more precise result, implying in particular that the faces $F_{Q}$ of $\mathcal{O}_{x}$ are themselves orbitopes under suitable groups.

Theorem 5.2. Let $x \in \mathfrak{p}$, let $F$ be a face of the orbitope $\mathcal{O}_{x}$.

(a) There exists a face $Q$ of $P_{x}$ and an element $g \in K$ such that $F=g F_{Q}$.

(b) If $Q^{\prime}$ is another face of $P_{x}$ with $F \subseteq g^{\prime} F_{Q^{\prime}}$ for some $g^{\prime} \in K$, then there exists $w \in W$ such that $w Q \subseteq Q^{\prime}$.

In particular, $Q \mapsto F_{Q}$ induces a bijective correspondence between $W$-orbits of faces of $P_{x}$ and $K$-orbits of faces of $\mathcal{O}_{x}$, compatible with inclusion of faces.

For the proof observe the following lemma:

Lemma 5.3. Let $x \in \mathfrak{p}$, let $Q$ be a face of $P_{x}$, and let $y \in P_{x}$ with $P_{y} \cap Q \neq \varnothing$. Then $w y \in Q$ for some $w \in W$.

Proof. We can assume $Q \neq P_{x}$. Since every face of a polytope is exposed, there is a supporting hyperplane $H \subseteq \mathfrak{a}$ of $P_{x}$ with $Q=H \cap P_{x}$. Since $P_{y} \subseteq P_{x}$ and $H \cap P_{y}$ is not empty, the hyperplane $H$ is a supporting hyperplane of $P_{y}$ as well. In particular, $Q^{\prime}=H \cap P_{y}$ is a face of $P_{y}$, and therefore contains an extreme point $y^{\prime}$ of $P_{y}$. Thus $y^{\prime} \in Q^{\prime} \subseteq H \cap P_{x}=Q$, and $y^{\prime}=w y$ for some $w \in W$ since $P_{y}=\operatorname{conv}(W y)$.

\subsection{PROOF of Theorem 5.2.}

(a) Let $F$ be a face of $\mathcal{O}_{x}$. By Theorem 4.2, $\mathcal{O}_{x}$ is a spectrahedron, so all faces are exposed. Hence there exist $z \in \mathfrak{p}$ and $c \in \mathbb{R}$ such that $H=\{y \in \mathfrak{p}:\langle y, z\rangle=c\}$ is a supporting hyperplane of $\mathcal{O}_{x}$ with $H \cap \mathcal{O}_{x}=F$. Upon replacing $F$ with $g F$ for some $g \in K$ we can assume $z \in \mathfrak{a}$, since $z$ is $K$-conjugate to an element of a. Then $H \cap \mathfrak{a}$ is a supporting hyperplane of $P_{x}$, and so $Q:=H \cap P_{x}$ is a face of $P_{x}$. Clearly $F=F_{Q}$.

(b) By (a) it suffices to show: If $Q, Q^{\prime}$ are faces of $P_{x}$, and if $g F_{Q} \subseteq F_{Q^{\prime}}$ for some $g \in K$, then there exists $w \in W$ with $w Q \subseteq Q^{\prime}$. Let $y \in \operatorname{relint}(Q)$. Since $Q \subseteq F_{Q}$ we have $g y \in F_{Q^{\prime}}$, and therefore $\pi(g y) \in Q^{\prime}$. On the other hand, $\pi(g y) \in \pi(K y)=P_{y}$. So Lemma 5.3 applies and shows $w y \in Q^{\prime}$ for some $w \in W$. Since $y \in \operatorname{relint}(Q)$, this implies $w Q \subseteq Q^{\prime}$.

In particular, if $Q, Q^{\prime}$ are faces of $P_{x}$ for which $F_{Q}$ and $F_{Q^{\prime}}$ are $K$-conjugate, then $Q$ and $Q^{\prime}$ are $W$-conjugate.

Recall that a face $Q$ of a polytope $P$ is called a facet if $\operatorname{dim}(Q)=\operatorname{dim}(P)-1$.

Corollary 5.5. The maximal proper faces of $\mathcal{O}_{x}$ are precisely the $K$-conjugates of the faces $F_{Q}$, where $Q$ is a facet of $P_{x}$. 
5.6. We apply this result to the study of the coorbitope $\mathcal{O}_{x}^{o}$. First recall the definition of the polar of a convex set. Let $V$ be a real vector space, $\operatorname{dim}(V)<\infty$. For any set $M \subseteq V$ let $M^{o}=\left\{l \in V^{\vee}: \forall x \in M l(x) \leq 1\right\}$, the polar set of $M$. Usually a euclidean inner product $\langle-,-\rangle$ on $V$ will be fixed, then we identify $M^{o}$ with the set $\{y \in V: \forall x \in M\langle x, y\rangle \leq 1\}$. If $M$ is compact and 0 is an interior point of $M$, the same holds for $M^{o}$.

If the compact group $K$ acts on $V$ and $x \in V$, the polar set of the orbitope $\mathcal{O}_{x}=\operatorname{conv}(K x)$ is called the coorbitope of $x$ [18]. Clearly the group $K$ acts on $\mathcal{O}_{x}^{o}$, but in general $\mathcal{O}_{x}^{o}$ won't be a $K$-orbitope. Below (Corollary 5.9 and Theorem 6.7) we'll identify those cases when this happens.

5.7. For any irreducible abstract root system $(V, \Sigma)$ (possibly non-reduced), the Weyl group $W$ acts irreducibly on $V$. For any $0 \neq x \in V$, the polytope $P_{x}=$ $\operatorname{conv}(W x)$ therefore contains an open neighborhood of the origin. If $(V, \Sigma)$ is not necessarily irreducible and $x \in V$, it follows that the polytope $P_{x}$ is full-dimensional if and only if every irreducible component of $\Sigma$ contains a root $\alpha$ with $\alpha(x) \neq 0$. Moreover in this case, 0 is an interior point of $P_{x}$.

Let $K \rightarrow S O(V)$ be a polar representation, and let $x \in V$. The previous discussion implies that when $V$ is irreducible, the origin is an interior point of $\mathcal{O}_{x}$ as soon as $x \neq 0$. When $V$ is an arbitrary polar representation of $K$, let $V=\bigoplus V_{i}$ be the decomposition into irreducible $K$-modules as in Remark 1.3, and let $x=$ $\sum_{i} x_{i} \in V$ with $x_{i} \in V_{i}$. Then $\mathcal{O}_{x}=\mathcal{O}_{x_{1}} \times \cdots \times \mathcal{O}_{x_{n}}$ by Remark 1.3. Therefore $\mathcal{O}_{x}$ is full-dimensional in $V$ iff 0 is an interior point of $\mathcal{O}_{x}$, and both are equivalent to $x_{i} \neq 0$ for each index $i$. It is also equivalent that $\pi(K x)=\mathcal{O}_{x} \cap \mathfrak{a}$ (Corollary 3.4) is full-dimensional in $\mathfrak{a}$.

When studying the orbitope $\mathcal{O}_{x}$, we can obviously assume that $\mathcal{O}_{x}$ is fulldimensional (or equivalently, 0 is an interior point of $\mathcal{O}_{x}$ ), by the previous discussion.

Proposition 5.8. Let $x \in \mathfrak{p}$ such that $\mathcal{O}_{x}$ is full-dimensional, and let $\mathcal{O}_{x}^{o} \subseteq \mathfrak{p}$ be the associated coorbitope. The $K$-orbits of extreme points of $\mathcal{O}_{x}^{o}$ are in natural bijective correspondence with the $W$-orbits of facets of the polytope $P_{x}$. In particular, $\mathcal{O}_{x}^{o}$ is the convex hull of finitely many $K$-orbits in $\mathfrak{p}$.

Proof. First recall the following general and easy fact (see [22, 2.1.4], for example). Let $\mathcal{O} \subseteq \mathbb{R}^{n}$ be any compact convex body which contains a neighborhood of 0 , and let $\mathcal{O}^{o} \subseteq \mathbb{R}^{n}$ be the convex body polar to $\mathcal{O}$. For any face $F$ of $\mathcal{O}$ let $\widehat{F}=\{y \in$ $\left.\mathcal{O}^{o}: \forall \bar{x} \in F\langle x, y\rangle=1\right\}$. Then $\widehat{F}$ is an exposed face of $\mathcal{O}^{\circ}$, and $F \mapsto \widehat{F}$ restricts to an inclusion-reversing bijection between exposed faces of $\mathcal{O}$ and exposed faces of $\mathcal{O}^{\circ}$, with inverse map $G \mapsto \widehat{G}$.

To prove the proposition we can assume $x \in C$. Let $\operatorname{Fac}(x)$ be a set of representatives of the $W$-orbits of facets of $P_{x}$. Let $z$ be an exposed extreme point of $\mathcal{O}_{x}^{o}=\{y \in \mathfrak{p}: \forall g \in K\langle g x, y\rangle \leq 1\}$, and write $G_{z}:=\widehat{z}=\left\{y \in \mathcal{O}_{x}:\langle y, z\rangle=1\right\}$. By the fact just recalled, $G_{z}$ is a maximal face of $\mathcal{O}_{x}$, and so $G_{z}=g F_{Q}$ for some $Q \in \operatorname{Fac}(x)$ and some $g \in K$ (Theorem 5.2, Corollary 5.5). It is easily checked that $G_{h z}=h G_{z}$ for any $h \in K$. If $u \in \mathcal{O}_{x}^{o}$ is another exposed extreme point, and if 
$G_{u}=h F_{Q}$ for some $h \in K$, then $G_{g h^{-1} u}=g h^{-1} G_{u}=G_{z}$, whence $g h^{-1} u=z$, so $u$ and $z$ are $K$-conjugate. This shows that the exposed extreme points of $\mathcal{O}_{x}^{o}$ consist of finitely many $K$-orbits, each of them corresponding to a different $W$-orbit of facets of $P_{x}$. Since exposed extreme points are dense within all extreme points (Straszewicz' theorem, e.g. $[22,1.4 .7])$, we conclude that all extreme points of $\mathcal{O}_{x}^{o}$ are exposed.

For each facet $Q$ of $P_{x}$ we claim conversely that $\widehat{F_{Q}}$ is an (exposed) extreme point of $\mathcal{O}_{x}^{o}$. Indeed, otherwise $\widehat{F_{Q}}$ would be a minimal exposed face of $\mathcal{O}_{x}^{o}$ of dimension $\geq 1$. But such a face cannot exist, since all extreme points of $\mathcal{O}_{x}^{o}$ are exposed. Altogether we have proved the bijection between $K$-orbits of extreme points of $\mathcal{O}_{x}^{o}$ and $W$-orbits of facets of $P_{x}$.

Corollary 5.9. Let $x \in \mathfrak{p}$. The coorbitope $\mathcal{O}_{x}^{o}$ is a $K$-orbitope itself if, and only if, all facets of the polytope $P_{x}$ are $W$-conjugate.

We will determine these cases explicitly in the next section, after having discussed the facets of $P_{x}$ in more detail.

Note that under the equivalent conditions of Corollary 5.9, the coorbitope $\mathcal{O}_{x}^{o}$ is a spectrahedron itself, by Theorem 4.2. In Sect. 7 we will uncover many more cases where this holds.

\section{Facets of the momentum polytope}

In the previous section, a close relation was established between the faces of the orbitope $\mathcal{O}_{x}$ and the faces of the momentum polytope $P_{x}$. The faces of the latter can be described in terms of root data. We start by recalling this description.

6.1. Let $(V, \Sigma)$ be an abstract root system (which may be non-reduced), fix an ordering $\leq$ on $V$, and let $\Gamma=\left\{\beta_{1}, \ldots, \beta_{n}\right\}$ be the corresponding system of simple positive roots. Let $\mu_{1}, \ldots, \mu_{n}$ be the dual basis of $\Gamma$ in $V$, so $\left\langle\beta_{i}, \mu_{j}\right\rangle=\delta_{i j}$ for $1 \leq i, j \leq n$. Let $W$ be the Weyl group, and let $C=\left\{x \in V:\left\langle\beta_{i}, x\right\rangle \geq 0\right.$, $1 \leq i \leq n\}$, the closed Weyl chamber associated to $\Gamma$.

Let $x \in C$ be a given point and write $P_{x}=\operatorname{conv}(W x)$. A subset $I \subseteq \Gamma$ is said to be $x$-connected if every connected component of $I$ contains a root $\beta$ with $\langle\beta, x\rangle \neq 0$. (Of course, connectedness notions refer to the Dynkin graph.) Let $W_{I}$ be the subgroup of $W$ generated by the root reflections $s_{\beta}$ where $\beta \in I$. The following result is quoted from Casselman [5, Theorem 3.1], where it is proved in the more general context of arbitrary finite Coxeter groups. As Casselman remarks, the result is already implicit in much older work of Satake [19] and Borel and Tits [3]. A related discussion can also be found in [2, §6] and [1, §4].

Theorem 6.2. Let $x \in C$. The map $I \mapsto \operatorname{conv}\left(W_{I} x\right)$ induces a bijection between the $x$-connected subsets $I$ of $\Gamma$ and the $W$-orbits of faces of $P_{x}=\operatorname{conv}(W x)$. For any such $I$ one has $\operatorname{dim} \operatorname{conv}\left(W_{I} x\right)=|I|$.

Here we are mainly interested in the facets of $P_{x}$. Assume that $P_{x}$ is fulldimensional in $V$, or equivalently, that every connected component of $\Gamma$ contains a root $\beta$ with $\langle\beta, x\rangle \neq 0$ (see 5.7). For facets the theorem gives: 
Corollary 6.3. Let $x \in C$ such that $P_{x}$ is full-dimensional, and let I $(x)$ denote the set of indices $i \in\{1, \ldots, n\}$ for which $\Pi \backslash\left\{\beta_{i}\right\}$ is $x$-connected. For $i \in I(x)$ let

$$
P_{x}(i):=\left\{y \in P_{x}: \mu_{i}(y)=\mu_{i}(x)\right\} .
$$

Then $P_{x}(i)$ is a facet of $P_{x}$. Conversely, every facet of $P_{x}$ is $W$-conjugate to $P_{x}(i)$ for a unique index $i \in I(x)$.

Proof. Let $i \in I(x)$. Clearly, $P_{x}(i)$ is a face of $P_{x}$, and is proper since $P_{x}$ is full-dimensional. Let $W^{\prime}:=W_{\Pi \backslash \beta_{i}}=\left\langle s_{\beta}: \beta \in \Pi, \beta \neq \beta_{i}\right\rangle$. Then $\operatorname{conv}\left(W^{\prime} x\right) \subseteq$ $P_{x}(i)$ holds since $\mu_{i}\left(s_{\beta_{j}}(y)\right)=\mu_{i}(y)$ for every $j \neq i$. By Theorem 6.2, $\operatorname{conv}\left(W^{\prime} x\right)$ is a facet of $P_{x}$, so we have equality. The remaining assertion follows directly from Theorem 6.2 as well.

We will also use the following (well-known) fact:

Lemma 6.4. Let $x \in C$ such that $P_{x}$ is full-dimensional. Then $\mu_{i}(x)>0$ for every $i=1, \ldots, n$.

Proof. It is enough to prove this in the case where the root system is simple and $0 \neq x \in C$. Since $\beta_{j}(x) \geq 0$ for all $j=1, \ldots, n$ and $\beta_{j}(x)>0$ for at least one $j$, the lemma follows from the fact that the inverse of the Cartan matrix has strictly positive coefficients [15].

6.5. Now again consider the adjoint representation of $K$ on $\mathfrak{p}$. We apply Corollary 6.3 to the system $\Sigma$ of restricted roots of $(\mathfrak{g}, \mathfrak{a})$. In this way we are going to identify explicitly the $K$-orbits of extreme points of the coorbitope $\mathcal{O}_{x}^{o}$ (see Proposition 5.8).

Let $x \in C \subseteq \mathfrak{a}$ such that $P_{x}$ is full-dimensional, and let $I(x)=\{i \in$ $\{1, \ldots, n\}: \Gamma \backslash\left\{\beta_{i}\right\}$ is $x$-connected $\}$ (as in Corollary 6.3). For every $i \in I(x)$ we have the facet $P_{x}(i)=\left\{y \in P_{x}: \mu_{i}(y)=\mu_{i}(x)\right\}$ of $P_{x}$. For easier notation, let us write $F_{i}$ instead of $F_{P_{x}(i)}=\left\{y \in \mathcal{O}_{x}: \pi(y) \in P_{x}(i)\right\}$. By Theorem 5.2, $F_{i}$ is a maximal proper face of $\mathcal{O}_{x}$, and every maximal proper face is $K$-conjugate to $F_{i}$ for a unique index $i \in I(x)$ (using also Corollary 6.3). Given $i \in I(x)$, there is a unique extreme point $z_{i}$ of $\mathcal{O}_{x}^{o}$ that corresponds to $F_{i}$ under polarity, characterized by $F_{i}=\widehat{z_{i}}=\left\{y \in \mathcal{O}_{x}:\left\langle y, z_{i}\right\rangle=1\right\}$ (see proof of Proposition 5.8). The points $z_{i}$, for $i \in I(x)$, represent the pairwise different $K$-orbits of extreme points in $\mathcal{O}_{x}^{o}$. Using Corollary 6.3 we identify these points as follows.

Let $i \in I(x)$, let $h_{\mu_{i}} \in \mathfrak{a}$ be the element satisfying $\left\langle h_{\mu_{i}}, y\right\rangle=\mu_{i}(y)$ for all $y \in \mathfrak{a}$. Note that $C$ is the cone generated by $h_{\mu_{1}}, \ldots, h_{\mu_{n}}$. By Lemma 6.4 we have $\mu_{i}(x)>0$. We claim that $z_{i}=h_{\mu_{i}} / \mu_{i}(x)$.

Indeed, the element $z:=h_{\mu_{i}} / \mu_{i}(x)$ satisfies $\langle y, z\rangle=\langle\pi(y), z\rangle=$ $\mu_{i}(\pi(y)) / \mu_{i}(x)$ for all $y \in \mathcal{O}_{x}$. Since $\pi(y) \in P_{x}$ (Corollary 3.4), this shows $\langle y, z\rangle \leq 1$, with equality if and only if $\pi(y) \in P_{x}(i)$. So we have proved:

Corollary 6.6. Let $x \in C$ such that $P_{x}$ is full-dimensional. The coorbitope $\mathcal{O}_{x}^{o} \subseteq \mathfrak{p}$ is the convex hull of the union of the $K$-orbits of the elements $z_{i}=h_{\mu_{i}} / \mu_{i}(x) \in C$, for $i$ running through $I(x)$. 
A particularly interesting case arises when $|I(x)|=1$, i.e. the polytope $P_{x}$ has only one $W$-orbit of facets. By Corollary 5.9 it is equivalent that the coorbitope $\mathcal{O}_{x}^{o}$ is a $K$-orbitope itself. We will say that $\mathcal{O}_{x}$ is a $(K-)$ biorbitope in this case, and we can characterize it as follows:

Theorem 6.7. Let $0 \neq x \in C$ such that $P_{x}$ is full-dimensional. Then $\mathcal{O}_{x}$ is a $K$ biorbitope if, and only if, the Lie algebra $\mathfrak{g}$ is simple, the restricted root system $\Gamma$ is not of type $D_{n}(n \geq 4)$ or $E_{n}(n=6,7,8)$, and the following holds: there is a simple restricted root $\beta \in \Gamma$ with $\gamma(x)=0$ for all $\gamma \in \Gamma \backslash\{\beta\}$, and such that $\Gamma \backslash\{\beta\}$ is connected.

In other words, the condition is saying that $\beta(x) \neq 0$ for only one simple restricted root $\beta$, that $\beta$ sits at an end of the restricted Dynkin graph $\Gamma$, and that $\Gamma$ has at most one other end. In 8.12 and 8.13 we'll make all biorbitopes explicit for the classical Lie algebras.

Proof. By Corollary 6.6, $\mathcal{O}_{x}$ is a biorbitope if and only if $P_{x}$ has only one $W$-orbit of facets. One sees immediately that this can hold only when the restricted root system $\Sigma$ is irreducible. Therefore we may assume that the Lie algebra $\mathfrak{g}$ is simple.

Let $\Gamma_{1}=\{\beta \in \Gamma: \beta(x) \neq 0\}$. We say that $\beta \in \Gamma$ is a boundary root if $\Gamma \backslash\{\beta\}$ is connected. If $\Gamma_{1}$ contains a non-boundary root then $P_{x}$ has two non-conjugate facets. Indeed, choose two different boundary roots $\beta_{i}$ and $\beta_{j}$. Then $P_{x}(i)$ and $P_{x}(j)$ are both facets of $P_{x}$, and are not $W$-conjugate, according to Corollary 6.3. Exactly the same argument works if $\Gamma_{1}$ contains two different boundary roots $\beta_{i}$, $\beta_{j}$.

So all facets of $P_{x}$ can only be $W$-conjugate if $\Gamma_{1}$ consists of just one single boundary root. Conversely, if this is the case then the conjugacy classes of facets of $P_{x}$ correspond precisely to the remaining boundary roots. This proves the equivalence in the theorem, since $D_{n}(n \geq 4)$ and $E_{n}(n=6,7,8)$ are precisely the simple root systems with more than two boundary roots.

Example 6.8. Let $n \geq 4$ and $\mathfrak{g}=\operatorname{so}(n, n)$, so $K=S O(n) \times S O(n)$. For the description of $\mathfrak{a}, C$, the $\alpha_{i}$ and $\lambda_{i}$ see Example 4.8. The restricted root system is of type $D_{n}$. If we take $x=(1, \ldots, 1)$ as in Remark 4.9, we have $\alpha_{i}(x)=0$ for all $i \neq n$, so $I(x)=\{n\}$ is a singleton set. Yet $P_{x}$ has two $W$-orbits of facets, represented by the facets $P_{x}(1)=\left\{y \in P_{x}: y_{1}=1\right\}$ and $P_{x}(n-1)=\left\{y \in P_{x}\right.$ : $\left.y_{1}+\cdots+y_{n-1}-y_{n}=n-2\right\}$. Hence the orbitope $\mathcal{O}_{x}=\operatorname{conv} S O(n)$ has two $K-$ orbits of maximal dimensional faces, a fact already proved in [18, Theorem 4.11]. This means that the coorbitope $\mathcal{O}_{x}^{o}=S O(n)^{o}$ is not an orbitope, rather

$$
S O(n)^{o}=\operatorname{conv}\left(K z_{1} \cup K z_{n-1}\right)
$$

by Corollary 6.6 , where $z_{1}=(1,0, \ldots, 0)$ and $z_{n-2}=\frac{1}{n-2}(1, \ldots, 1,-1)$ (notation as in Example 4.8 and Remark 4.9). A similar remark applies when $x=(1,0, \ldots, 0)$ (here $\mathcal{O}_{x}$ is the unit ball of the nuclear norm on $M_{n}(\mathbb{R})$, see Example 8.4) and of $x=(1, \ldots, 1,-1)$ (here $\mathcal{O}_{x}$ is the convex hull of $O^{-}(n)$, which is of course linearly isomorphic to conv $S O(n)$ ). 
Remarkably, whenever $\mathcal{O}_{x}$ is a biorbitope, the coorbitope $\mathcal{O}_{x}^{o}$ is a positive scaling of $\mathcal{O}_{x}$ :

Theorem 6.9. Let $\mathfrak{g}$ be simple and $0 \neq x \in C$, and assume that $\mathcal{O}_{x}$ is a biorbitope, i.e. $|I(x)|=1$. Then there is a real number $c>0$ such that $\mathcal{O}_{x}^{o}=c \cdot \mathcal{O}_{x}$.

Proof. We have $I(x)=\{i\}$ where $\beta_{i} \in \Gamma$ is a boundary root (Theorem 6.7). By Corollary 6.6, $\mathcal{O}_{x}^{o}$ is the convex hull of $K z_{i}$ where $z_{i}=h_{\mu_{i}} / \mu_{i}(x)$. The element $x$ itself is a scalar multiple of $h_{\mu_{i}}$ since $\beta_{j}(x)=0$ for all $\beta_{j} \in \Gamma \backslash\left\{\beta_{i}\right\}$. More precisely $x=\beta_{i}(x) h_{\mu_{i}}$, since both elements give the same value under every $\beta_{j}$. This implies $\mu_{i}(x)=\beta_{i}(x) \mu_{i}\left(h_{\mu_{i}}\right)=\beta_{i}(x)\left|\mu_{i}\right|^{2}$. So $z_{i}=h_{\mu_{i}} / \mu_{i}(x)=x / \beta_{i}(x)^{2}\left|\mu_{i}\right|^{2}$, and therefore

$$
\mathcal{O}_{x}^{o}=\frac{1}{\beta_{i}(x)^{2} \cdot\left|\mu_{i}\right|^{2}} \mathcal{O}_{x}
$$

\section{Doubly spectrahedral orbitopes}

Following Saunderson et al. [20] we use the term doubly spectrahedral convex sets to refer to convex sets $S$ in $\mathbb{R}^{n}$ for which both $S$ and the polar convex set $S^{o}$ are spectrahedra. As remarked in [20], it is a very special phenomenon that the polar set of a spectrahedron is again a spectrahedron. Apart from polyhedra (which have this property for obvious reasons) it seems that only one other distinct family of doubly spectrahedral convex sets is known, namely the homogeneous convex cones (Vinberg [25] and Chua [6], see [20, 6.1]). In addition, the convex hull of the matrix group $S O(n)$ is doubly spectrahedral for every $n \geq 1$, by the main theorem of [20]. In fact, explicit spectrahedral representations for both conv $S O(n)$ and $S O(n)^{o}$ were constructed in [20].

Below we show that all polar orbitopes $\mathcal{O}_{x}$ with "rational coordinates" are doubly spectrahedral as well. Moreover we'll give explicit linear matrix inequality representations for those orbitopes and their polars. As a particular case, we recover the results from [20], see Example 7.7 below.

Let $\mathfrak{g}=\mathfrak{k} \oplus \mathfrak{p}$ be a real semisimple Lie algebra with Cartan decomposition, and consider the adjoint representation of $K$ on $\mathfrak{p}$ as before. We use notation and conventions from Sect. 2. In particular, $\mathfrak{a}$ is a maximal abelian subspace of $\mathfrak{p}$, and $C \subseteq \mathfrak{a}$ is the Weyl chamber with respect to the fixed ordering on $\mathfrak{a}$. As before, let $\Gamma=\left\{\beta_{1}, \ldots, \beta_{n}\right\} \subseteq \mathfrak{a}^{\vee}$ be the simple positive restricted roots.

Definition 7.1. Given $x \in \mathfrak{a}$, we say that the $K$-orbitope $\mathcal{O}_{x}=\operatorname{conv}(K x)$ has rational coordinates if there is $b \in \mathbb{R}$ such that $\beta_{j}(x) \in \mathbb{Q} b$ for $j=1, \ldots, n$.

Since any two choices of $\mathfrak{a} \subseteq \mathfrak{p}$ are conjugate under $K[11,6.51]$, and since every $K$-orbit in $\mathfrak{p}$ intersects $\mathfrak{a}$ in one full $W$-orbit (Corollary 3.5 ), the property of having rational coordinates depends only on the orbit $K x$, and neither on the choice of $\mathfrak{a}$ nor on the particular choice of a representative of $K x$ in $\mathfrak{a}$. 
Theorem 7.2. Let $\mathcal{O}_{x}$ be a polar orbitope with rational coordinates. Then both $\mathcal{O}_{x}$ and $\mathcal{O}_{x}^{o}$ are spectrahedra.

For $\mathcal{O}_{x}$, a spectrahedral representation has been given in Theorem 4.4. In Remark 7.6 below we explain how to find one for $\mathcal{O}_{x}^{o}$. Explicit descriptions of these orbitopes are contained in the next section, c.f. Remark 8.9.

Given $x \in \mathfrak{a}$, let $P_{x}=\operatorname{conv}(W x)$ be the momentum polytope of $x$ as before, and let $P_{x}^{o}$ be the polar set of $P_{x}$ in $\mathfrak{a}$, i.e.

$$
P_{x}^{o}=\{y \in \mathfrak{a}: \forall w \in W\langle w x, y\rangle \leq 1\}
$$

We have the following lemma:

Lemma 7.3. If $x \in C$ then $P_{x}^{o} \cap C=\{y \in C:\langle x, y\rangle \leq 1\}$.

Proof. If $y \in C$ then $\langle w x, y\rangle \leq\langle x, y\rangle$ for every $w \in W$ [13, Lemma 3.2]. Therefore, if $\langle x, y\rangle \leq 1$ then $y \in P_{x}^{o}$. The opposite inclusion is trivial from the definition.

Recall that $\pi: \mathfrak{p} \rightarrow \mathfrak{a}$ denotes orthogonal projection to $\mathfrak{a}$. Forming the polar convex body commutes with projection to (or intersection with) $\mathfrak{a}$ :

Lemma 7.4. Let $\mathcal{O} \subseteq \mathfrak{p}$ be a $K$-invariant convex set, and let $Q:=\mathcal{O} \cap \mathfrak{a}=\pi(\mathcal{O})$ (3.4). Then $\pi\left(\mathcal{O}^{o}\right)=\mathcal{O}^{o} \cap \mathfrak{a}=Q^{o}$ (the polar set of $Q$ in $\mathfrak{a}$ ).

Proof. For $y \in \mathfrak{a}$ and $z \in \mathfrak{p}$ we have $\langle y, \pi(z)\rangle=\langle y, z\rangle$. From this the lemma follows immediately.

7.5. ProOF of Theorem 7.2. We can assume $x \in C$. Since $x$ has rational coordinates we can assume $\beta_{j}(x) \in \mathbb{Q}$ for $j=1, \ldots, n$, after scaling $x$ with a suitable positive real number. So there exist rational numbers $c_{j} \geq 0$ such that $\langle x, y\rangle=\sum_{j=1}^{n} c_{j} \mu_{j}(y)$ for all $y \in \mathfrak{a}$ (namely $c_{j}=\beta_{j}(x)$ ). Hence, and by Lemma 2.9, there are an integer $k \geq 1$ and an integral dominant weight $\omega \in \mathfrak{h}^{\vee}$ of $\left(\mathfrak{g}_{\mathbb{C}}, \mathfrak{h}_{\mathbb{C}}\right)$ such that $k \cdot\langle x, y\rangle=\omega(y)$ for all $y \in \mathfrak{a}$. By the highest weight theorem, there is an irreducible representation $\rho$ of $\mathfrak{g}_{\mathbb{C}}$ with highest weight $\omega$. From Lemmas 7.3 and 7.4 we get

$$
\mathcal{O}_{x}^{o} \cap C=P_{x}^{o} \cap C=\{y \in C:\langle x, y\rangle \leq 1\}
$$

We claim that (4) implies

$$
\mathcal{O}_{x}^{o}=\{y \in \mathfrak{p}: \rho(y) \preceq k \cdot \mathrm{id}\} .
$$

Indeed, both sets in (5) are $K$-invariant, so it suffices to check that their intersections with $C$ coincide. For $y \in C$ the largest eigenvalue of $\rho(y)$ is $\omega(y)=k\langle x, y\rangle$ [Lemma 4.6(b)]. So (5) follows indeed from (4), and the theorem is proved. 
Remark 7.6. The highest weights of irreducible representations of $\left(\mathfrak{g}_{\mathbb{C}}, \mathfrak{h}_{\mathbb{C}}\right)$ are the nonnegative integral linear combinations of the fundamental weights $\omega_{i}=\frac{1}{2}\left|\alpha_{i}\right|^{2} \lambda_{i}$ $(i=1, \ldots, l)$. The restriction of $\omega_{i} \in \mathfrak{h}^{\vee}$ to $\mathfrak{a}$ is 0 if $r\left(\alpha_{i}\right)=0$, and is $\frac{1}{2 m}\left|\alpha_{i}\right|^{2} \mu_{j}$ if $r\left(\alpha_{i}\right)=\beta_{j} \neq 0$, where $m \in\{1,2\}$ is the number of simple roots in $\Pi$ that restrict to $\beta$ (Lemma 2.9). Since the $\left|\alpha_{i}\right|^{2}$ are explicit rational numbers, we see how to find, for given $x \in \mathfrak{a}$ with rational coordinates, a real number $c>0$ and an integral dominant weight $\omega$ of $\left(\mathfrak{g}_{\mathbb{C}}, \mathfrak{h}_{\mathbb{C}}\right)$ such that $\langle c x, y\rangle=\omega(y)$ for all $y \in \mathfrak{a}$.

Example 7.7. We illustrate the previous remark with the example already studied in Example 4.8, so consider the action of $K=S O(n) \times S O(n)$ on $M_{n}(\mathbb{R})$ for $n \geq 3$. We take the identity matrix $x=I_{n}$ as in Remark 4.9 and are looking for a linear matrix inequality description of the coorbitope $\mathcal{O}_{x}^{o}=(\operatorname{conv} S O(n))^{o}$. The orbitope $\mathcal{O}_{x}$ has rational coordinates since $\alpha_{i}(x)=0$ for $1 \leq i<n$ and $\alpha_{n}(x)=2$ (see Example 4.8). Since $\langle x, y\rangle=\sum_{i=1}^{n} y_{i}=2 \lambda_{n}(y)$ for $y \in \mathfrak{a}$, the procedure in Remark 7.6 leads to the spectrahedral representation

$$
(\operatorname{conv} S O(n))^{o}=\left\{y \in M_{n}(\mathbb{R}): \rho_{n}\left(\begin{array}{cc}
0 & y \\
y^{t} & 0
\end{array}\right) \preceq \frac{1}{2} \mathrm{id}\right\}
$$

where $\rho_{n}$ is the $n$th fundamental representation. This is in accordance with Saunderson et al. [20, Theorem 1.1], c.f. the remark in Remark 4.9.

For $n=3, \mathcal{O}_{x}^{o}$ is a $K$-orbitope itself. For $n \geq 4, \mathcal{O}_{x}^{o}$ is the convex hull of two $K$-orbits, but not of one (Example 6.8).

\section{Examples}

We describe all irreducible polar representations that arise from semisimple Lie algebras of classical type. Roughly, these are the well-known unitary group actions on rectangular matrices, and on (skew-) hermitian resp. (skew-) symmetric square matrices, over $\mathbb{K}=\mathbb{R}, \mathbb{C}$ or $\mathbb{H}$, where $\mathbb{H}$ is the skew-field of Hamilton quaternions. (For $\mathbb{K}=\mathbb{H}$ there is no action on (skew-) symmetric matrices.) In each case we mention a standard choice of a maximal abelian subspace $\mathfrak{a}$ and of a Weyl chamber C. Using Kostant's results, in particular Proposition 3.2 and Corollary 3.4, this allows us to give explicit descriptions of the respective orbitopes in all cases. Naturally, this uses the description of the (reduced) root systems and of the fundamental weights, for which there are many references (e.g. [11,16,24]). We will see that the corresponding orbitopes can be described in terms of Ky Fan norm balls, which in turn are defined using singular values of matrices.

8.1. First recall the singular value decomposition. Let always $\mathbb{K}$ be one of $\mathbb{R}, \mathbb{C}$ or $\mathbb{H}$, and let $U(n, \mathbb{K})$ denote the unitary group over $\mathbb{K}$, i.e. $U(n, \mathbb{K})=\{g \in$ $\left.M_{n}(\mathbb{K}): g g^{*}=I_{n}\right\}$ where $g^{*}=\bar{g}^{t}$. So $U(n, \mathbb{R})=O(n)$ is the real orthogonal group, $U(n, \mathbb{C})=U(n)$ is the usual (complex) unitary group and $U(n, \mathbb{H})=\operatorname{Sp}(n)$ is the symplectic group. Given a (rectangular) matrix $x \in M_{m \times n}(\mathbb{K})$ where $m \geq n$, there exist unitary matrices $u \in U(m, \mathbb{K})$ and $v \in U(n, \mathbb{K})$, together with real numbers $a_{1} \geq \cdots \geq a_{n} \geq 0$, such that $u x v^{*}$ is the $m \times n$ matrix whose upper $n$ rows are $\operatorname{diag}\left(a_{1}, \ldots, a_{n}\right)$ and whose lower $m-n$ rows are zero. The $a_{i}$ are uniquely 
determined by $x$, they are called the singular values of $x$ and denoted $\sigma_{i}(x):=a_{i}$ $(1 \leq i \leq n)$. For $\mathbb{K}=\mathbb{R}$ or $\mathbb{C}$ this is classical (e.g. [10, 2.6.3]), here $a_{1}^{2}, \ldots, a_{n}^{2}$ are the eigenvalues of the psd hermitian matrix $x^{*} x$. For $\mathbb{K}=\mathbb{H}$, essentially the same is true (with eigenvalues replaced by right eigenvalues), but less well-known; see $[26,7.2]$ and $[9,5.7]$ for details.

8.2. Let $\mathbb{K}=\mathbb{R}, \mathbb{C}$ or $\mathbb{H}$, and let $V=M_{m \times n}(\mathbb{K})$ with $m \geq n$. For $k=1, \ldots, n$ and $x \in V$ let

$$
\|x\|_{k}:=\sigma_{1}(x)+\cdots+\sigma_{k}(x),
$$

sum of the $k$ largest singular values of $x$. This defines a matrix norm on the space of matrices, the kth Ky Fan norm ([8] and [10, 7.4.8 and 7.4.10]). In particular, all balls with respect to any of these norms are convex. Note that the first Ky Fan norm $\|x\|_{1}=\sigma_{1}(x)$ is the operator norm of $x$. The last one $\|x\|_{n}=\sum_{i=1}^{n} \sigma_{i}(x)$ is called the nuclear norm and often denoted $\|x\|_{*}$.

In view of Remark 1.3, we restrict our discussion of classical polar orbitopes to orbitopes that arise from simple real Lie algebras $\mathfrak{g}$ of classical type.

Example 8.3. Let $m \geq n \geq 1$. Consider $K=S O(m) \times S O(n)$ (case $\mathbb{K}=\mathbb{R})$ resp. $K=S(U(m) \times U(n))($ case $\mathbb{K}=\mathbb{C})$ resp. $K=S p(m) \times S p(n)($ case $\mathbb{K}=\mathbb{H})$, together with the action of $(u, v) \in K$ on $x \in V=M_{m \times n}(\mathbb{K})$ by $u x v^{*}$. This is a polar representation, arising from the simple Lie algebra $\mathfrak{g}=\operatorname{so}(m, n)$ resp. $\mathfrak{g}=\operatorname{su}(m, n)$ resp. $\mathfrak{g}=\operatorname{sp}(m, n)($ assume $m+n \neq 2,4$ if $\mathbb{K}=\mathbb{R})$. For $\mathfrak{a} \cong \mathbb{R}^{n}$ we can take the space of real matrices that are diagonal in the upper $n$ rows and zero below. If $\left(a_{1}, \ldots, a_{n}\right)$ is the diagonal part of such a matrix $x$, let us write $x_{i}:=a_{i}$. The case $\mathbb{K}=\mathbb{R}$ and $\mathfrak{g}=\operatorname{so}(m, n)$ with $m=n$ is exceptional (see Example 8.4 below), so let us first discard it. In all other cases the Weyl chamber $C$ consists of all $x \in \mathfrak{a}$ with $x_{1} \geq \cdots \geq x_{n} \geq 0$. Moreover, using Proposition 3.2 we see that

$$
P_{x} \cap C=\left\{y \in C: y_{1}+\cdots+y_{k} \leq x_{1}+\cdots+x_{k}(k=1, \ldots, n)\right\}
$$

If $x \in C$ then clearly $x_{i}=\sigma_{i}(x)$, the $i$ th singular value of $x$. It follows for arbitrary $x \in V$ that

$$
\mathcal{O}_{x}=\left\{y \in V:\|y\|_{k} \leq\|x\|_{k}(k=1, \ldots, n)\right\}
$$

since both sets are $K$-invariant and their intersections with $C$ coincide. So $\mathcal{O}_{x}$ is an intersection of balls with center 0 with respect to the Ky Fan norms $\|\cdot\|_{k}$ $(k=1, \ldots, n)$, the radii of the balls being the norms of $x$. Note that the $K$-orbit $K x$ consists of all matrices with the same singular values as $x$.

Example 8.4. Now consider the exceptional case $\mathbb{K}=\mathbb{R}$ and $m=n$ of the previous example, so we have the natural action of $K=S O(n) \times S O(n)$ on $V=M_{n}(\mathbb{R})$. Here the Weyl chamber $C$ consists of all $x=\operatorname{diag}\left(x_{1}, \ldots, x_{n}\right) \in \mathbb{R}^{n}$ with $x_{1} \geq$ $\cdots \geq x_{n-1} \geq\left|x_{n}\right|$, and

$$
P_{x} \cap C=\left\{y \in C: \sum_{i=1}^{k}\left(x_{i}-y_{i}\right) \geq 0(k=1, \ldots, n-1),\left|x_{n}-y_{n}\right| \leq \sum_{i=1}^{n-1}\left(x_{i}-y_{i}\right)\right\}
$$


Now the last diagonal entry $x_{n}$ of $x \in C$ coincides with the smallest singular value $\sigma_{n}(x)$ only up to sign. With similar reasoning as in Example 8.3 we conclude

$$
\mathcal{O}_{x}=\bigcap_{k=1}^{n}\left\{y:\|y\|_{k} \leq\|x\|_{k}\right\} \cap\left\{y: \sigma_{n}(x)-\sigma_{n}(y) \leq\|x\|_{n-1}-\|y\|_{n-1}\right\} .
$$

Remark 8.5. (Orthogonal vs. special orthogonal group) Let $m \geq n$, let $K=$ $S O(m) \times S O(n)$ and $K^{\prime}=O(m) \times O(n)$. If $m>n$, it is easy to see that the $K^{\prime}$-orbit of an arbitrary matrix $x \in M_{m \times n}(\mathbb{R})$ coincides with the $K$-orbit of $x$. For $m=n$ this is true if $\operatorname{det}(x)=0$, but otherwise $K^{\prime} x$ is the union of two distinct $K$-orbits, as one sees from the determinant. A spectrahedral representation of the $K^{\prime}$-orbitope $\mathcal{O}_{x}^{\prime}:=\operatorname{conv}\left(K^{\prime} x\right)$ was given in [18, Theorem 4.7] in the case $m=n$ (and $\mathcal{O}_{x}^{\prime}$ was called a Fan orbitope there). Note that $\mathcal{O}_{x}^{\prime}=\operatorname{conv} O(n)$ if $x=I_{n}$. Moreover, a spectrahedral representation of the coorbitope (conv $O(n))^{o}$ was provided in [18, (Corollary 4.9)]. Both representations can easily be derived from our discussion of $K$-(co)orbitopes.

Example 8.6. Next consider the actions of the classical compact simple Lie groups on hermitian matrices. Let $n \geq 2$, and let $K=S O(n)$ (case $\mathbb{K}=\mathbb{R}$ ) resp. $K=S U(n)($ case $\mathbb{K}=\mathbb{C})$ resp. $K=S p(n)$ (case $\mathbb{K}=\mathbb{H})$. Let $V=\{x \in$ $\left.M_{n}(\mathbb{K}): x=x^{*}\right\}$, the space of hermitian $n \times n$-matrices over $\mathbb{K}$, and let $V_{0}=$ $\{x \in V: \operatorname{tr}(x)=0\}$, where for $\mathbb{K}=\mathbb{H}$ the trace condition has to be replaced by $\operatorname{trd}(x)=0$ (reduced trace). We let $g \in K$ act on $x \in V$ by $g x g^{*}$. Clearly $V_{0}$ is $K$-invariant, and $V=V_{0} \oplus \mathbb{R}$ as $K$-modules. The action of $K$ on $V_{0}$ is an irreducible polar representation, resulting from the simple Lie algebra $\mathfrak{g}=\operatorname{sl}(n, \mathbb{R})$ resp. $\mathfrak{g}=s u(n, \mathbb{C})$ resp. $\mathfrak{g}=\operatorname{sl}(n, \mathbb{H})$. We let $\mathfrak{a} \cong \mathbb{R}^{n-1}$ be the space of all real diagonal matrices $x=\left(x_{1}, \ldots, x_{n}\right)$ with trace zero. The Weyl chamber is $C=\left\{x \in \mathfrak{a}: x_{1} \geq \cdots \geq x_{n}\right\}$, and for $x=\left(x_{1}, \ldots, x_{n}\right) \in \mathfrak{a}$ we have

$$
P_{x} \cap C=\left\{y \in C: \sum_{i=1}^{k} y_{i} \leq \sum_{i=1}^{k} x_{i}(i=1, \ldots, n)\right\}
$$

The $K$-orbit $K x$ consists of all hermitian matrices with the same eigenvalues as $x$ (for $\mathbb{K}=\mathbb{H}$ one has to speak of right eigenvalues instead of eigenvalues [9]).

In order to describe the $K$-orbitope $\mathcal{O}_{x}$ we replace $x \in V_{0}$ by $x^{\prime}=x+c I \in V$, where $c \geq 0$ is chosen such that $x^{\prime} \succeq 0$, i.e. $x^{\prime}$ is positive semidefinite ( $\left.p s d\right)$. Of course this doesn't change the orbitope up to an affine-linear isomorphism. So let $x \in V$ be a psd hermitian matrix. Then clearly $y \succeq 0$ holds for every $y \in \mathcal{O}_{x}$, and the sequence $\sigma(y)=\left(\sigma_{1}(y), \ldots, \sigma_{n}(y)\right)$ of singular values coincides with the sequence of (right) eigenvalues for these $y$. So we see that

$$
\mathcal{O}_{x}=\{y \in V: y \succeq 0, \sigma(y) \unlhd \sigma(x)\},
$$

where for nonincreasing sequences $a, b \in \mathbb{R}^{n}$ the majorization relation $\unlhd$ is defined by

$$
\left(b_{1}, \ldots, b_{n}\right) \unlhd\left(a_{1}, \ldots, a_{n}\right): \Leftrightarrow \sum_{i=1}^{k} b_{i} \leq \sum_{i=1}^{k} a_{i}
$$


for $k=1, \ldots, n$, with equality for $k=n[10,4.3 .41]$. In terms of Ky Fan norms this says that $\mathcal{O}_{x}$ is the set of all $y \in V$ with $y \succeq 0$ and $\|y\|_{k} \leq\|x\|_{k}$ for $k=1, \ldots, n-1$ and $\|y\|_{n}=\|x\|_{n}$ (provided that $x \succeq 0$ ).

Example 8.7. Next let the unitary group over $\mathbb{K}$ act on skew-hermitian matrices over $\mathbb{K}$ by $g x g^{*}$. For $\mathbb{K}=\mathbb{C}$ this is essentially the action of $S U(n)$ on hermitian matrices, already considered in Example 8.6, since a complex matrix $x$ is skewhermitian if and only if $i x$ is hermitian. For the remaining two cases we have $\mathbb{K}=\mathbb{R}$ and $K=S O(n)$ (with $n \geq 3$ ), or $K=\mathbb{H}$ and $K=S p(n)$ (with $n \geq 1$ ), and $V$ is the space of skew-hermitian $\left(x+x^{*}=0\right)$ matrices over $\mathbb{K}$ of size $n$. This is an irreducible polar representation of $K$, namely the adjoint action of $K$ on its Lie algebra $V=\operatorname{Lie}(K)$ (so $\mathfrak{g}=\operatorname{Lie}(K) \mathbb{C}$ here.) A maximal abelian subspace can be described as follows. If $\mathbb{K}=\mathbb{R}$ and $K=S O(n)$, put $m:=\left\lfloor\frac{n}{2}\right\rfloor$ and let $\mathfrak{a}$ consist of all real block matrices $x=\left(\begin{array}{cc}0 & \tilde{x} \\ -\tilde{x} & 0\end{array}\right)$ where $\tilde{x}=\operatorname{diag}\left(x_{1}, \ldots, x_{m}\right)$; if $n$ is odd, an extra row (at the bottom) and column (at the right) of zeros has to be added. If $\mathbb{K}=\mathbb{H}$ and $K=\operatorname{Sp}(n)$, let $\mathfrak{a}$ consist of all diagonal matrices $x=\left(i x_{1}, \ldots, i x_{n}\right)$ with $x_{1}, \ldots, x_{n} \in \mathbb{R}$.

To describe Weyl chamber and orbitopes, let first $\mathbb{K}=\mathbb{R}$ and $K=S O(n)$. The Weyl chamber $C$ consists of all $x \in \mathfrak{a}$ with $x_{1} \geq \cdots \geq x_{m} \geq 0$ (case $n$ odd), resp. $x_{1} \geq \cdots \geq x_{m-1} \geq\left|x_{m}\right|$ (case $n$ even). The description of $P_{x} \cap C$, for $x \in C$, is analogous to Example 8.3 resp. Example 8.4. The singular values of $x \in C$ are $x_{1}, x_{1}, \ldots, x_{m}, x_{m}$, with an extra zero if $n$ is odd. So we get $\mathcal{O}_{x}=\bigcap_{k=1}^{m}\{y \in$ $\left.V:\|y\|_{2 k} \leq\|x\|_{2 k}\right\}$ for $n=2 m+1$ odd, and

$$
\mathcal{O}_{x}=\bigcap_{k=1}^{m}\left\{y:\|y\|_{2 k} \leq\|x\|_{2 k}\right\} \cap\left\{y: \sigma_{n}(x)-\sigma_{n}(y) \leq\|x\|_{n-2}-\|y\|_{n-2}\right\}
$$

for $n=2 m$ even. Note that in either case, only the even Ky Fan norms are needed.

If $\mathbb{K}=\mathbb{H}$ and $K=S p(n)$, the Weyl chamber $C$ consists of all $x \in \mathfrak{a}$ with $x_{1} \geq \cdots \geq x_{m} \geq 0$, and we find again $\mathcal{O}_{x}=\bigcap_{k=1}^{n}\left\{y \in V:\|y\|_{k} \leq\|x\|_{k}\right\}$ for $x \in V$.

Example 8.8. There remains the action of the complex unitary group $K=U(n)$ on $V=\operatorname{sym}(n, \mathbb{C})$ resp. $V=\operatorname{so}(n, \mathbb{C})$ (symmetric resp. skew-symmetric complex matrices) by $g x g^{t}(g \in K, x \in V)$. Again this is an irreducible polar representation that arises from the simple Lie algebra $\mathfrak{g}=\operatorname{sp}(n, \mathbb{R})($ for $V=\operatorname{sym}(n, \mathbb{C}))$ resp. $\mathfrak{g}=s o^{*}(2 n)($ for $V=\operatorname{so}(n, \mathbb{C}))$.

First let $V=\operatorname{sym}(n, \mathbb{C})$. A maximal abelian subspace $\mathfrak{a}$ consists of all real diagonal matrices $x=\operatorname{diag}\left(x_{1}, \ldots, x_{n}\right)$, and the Weyl chamber is $C=\{x \in$ $\left.\mathfrak{a}: x_{1} \geq \cdots \geq x_{n} \geq 0\right\}$. For $x \in C$ we have $P_{x} \cap C=\left\{y \in C: y_{1}+\cdots+y_{k} \leq\right.$ $\left.x_{1}+\cdots+x_{k}(k=1, \ldots, n)\right\}$. Since $x_{i}=\sigma_{i}(x)$ for $x \in \mathfrak{a}$, we get

$$
\mathcal{O}_{x}=\left\{y \in \operatorname{sym}(n, \mathbb{C}):\|y\|_{k} \leq\|x\|_{k}(k=1, \ldots, n)\right\}
$$

In the skew-symmetric case $V=\operatorname{so}(n, \mathbb{C})$ let $m=\left\lfloor\frac{n}{2}\right\rfloor$. For $n$ even, a maximal abelian subspace $\mathfrak{a}$ consists of all block matrices $x=\left(\begin{array}{cc}0 & \tilde{x} \\ -\tilde{x} & 0\end{array}\right)$ with 
$\tilde{x}=\operatorname{diag}\left(x_{1}, \ldots, x_{m}\right)$ a real diagonal matrix. For $n$ odd the description is the same, except that one row (at the bottom) and one column (at the right) of zeros has to be added. In either case the Weyl chamber $C$ consists of all $x \in \mathfrak{a}$ with $x_{1} \geq \cdots \geq x_{m} \geq 0$. For $x \in \mathfrak{a}$ the singular values of $x$ are $x_{1}, x_{1}, \ldots, x_{m}, x_{m}$, together with an extra zero if $n$ is odd. Once more we therefore find

$$
\mathcal{O}_{x}=\left\{y \in \operatorname{so}(n, \mathbb{C}):\|y\|_{k} \leq\|x\|_{k}(k=1, \ldots, n)\right\}
$$

The fact that any (skew-) symmetric complex matrix is unitarily congruent to a real matrix in $\mathfrak{a}$ as above is known as Youla's theorem (see e.g. [10, Theorem 4.4.9]).

Remark 8.9. For all the examples from 8.3-8.8, the following is true: The orbitope $\mathcal{O}_{x}$ is doubly spectrahedral, provided that all singular values of $x$ are rational numbers. This follows from Theorem 7.2.

Remark 8.10. As we have seen, most of the classical irreducible polar orbitopes are intersections of Ky Fan balls of matrices, possibly intersected with suitable linear spaces of matrices (like (skew-) symmetric or (skew-) hermitian). It is easy to see that every Ky Fan ball is a spectrahedron. This gives a second proof of Theorem 4.2 in those cases where $\mathcal{O}_{x}$ is an intersection of such balls:

Proposition 8.11. Let $\mathbb{K} \in\{\mathbb{R}, \mathbb{C}, \mathbb{H}\}$ and $m \geq n$, let $1 \leq k \leq n$, and let $\|\cdot\|_{k}$ denote the kth Ky Fan norm on $M_{m \times n}(\mathbb{K})$ (8.2). Then the unit ball

$$
B_{k}:=\left\{x \in M_{m \times n}(\mathbb{K}):\|x\|_{k} \leq 1\right\}
$$

is a spectrahedron.

Proof. Let $A \in M_{N}(\mathbb{C})$ be a complex matrix with eigenvalues $\theta_{1}, \ldots, \theta_{N}$. The $k$ th exterior power $\Lambda^{k} A$ of $A$ is a square matrix of size $\left(\begin{array}{c}N \\ k\end{array}\right)$ that depends linearly on $A$, and whose eigenvalues are the sums $\theta_{i_{1}}+\cdots+\theta_{i_{k}}$ with $1 \leq i_{1}<\cdots<i_{k} \leq N$.

If $\mathbb{K}=\mathbb{H}$, we replace quaternions with complex $2 \times 2$ matrices, to avoid the problem of defining exterior powers of quaternion matrices. Let $A \in M_{N}(\mathbb{H})$ be hermitian $\left(A=A^{*}\right)$, with (real) right eigenvalues $\theta_{1}, \ldots, \theta_{N}$. Write $A=A_{1}+j A_{2}$ with $A_{1}, A_{2} \in M_{N}(\mathbb{C})$, and let

$$
\tilde{A}=\left(\begin{array}{cc}
A_{1} & -\bar{A}_{2} \\
A_{2} & \bar{A}_{1}
\end{array}\right)
$$

Then $\tilde{A}$ is a complex hermitian matrix of size $2 N$ with eigenvalues $\theta_{1}, \theta_{1}, \ldots, \theta_{N}, \theta_{N}$. Let us, for this purpose, define $\Lambda^{k} A$ to be the complex matrix $\Lambda^{k} \tilde{A}$ (of size $\left(\begin{array}{c}2 N \\ k\end{array}\right)$ ).

Now let $\mathbb{K}$ be any of $\mathbb{R}, \mathbb{C}, \mathbb{H}$, let $x \in M_{m \times n}(\mathbb{K})$, and let $\widehat{x} \in M_{m+n}(\mathbb{K})$ be the hermitian (block) matrix

$$
\widehat{x}=\left(\begin{array}{cc}
0 & x \\
x^{*} & 0
\end{array}\right)
$$

The (right) eigenvalues of $\widehat{x}$ are $\pm \sigma_{i}(x), i=1, \ldots, n$, together with $m-n$ additional zeros. It follows that $x \in B_{k}$, i.e. $\|x\|_{k} \leq 1$, if and only if all eigenvalues of $\Lambda^{k} \widehat{x}$ are $\leq 1$. In other words, this shows that $B_{k}$ is described by the linear matrix inequality

$$
B_{k}=\left\{x \in M_{m \times n}(\mathbb{K}): \Lambda^{k} \widehat{x} \preceq I\right\} .
$$


8.12. Finally, we record the cases when the orbitope $\mathcal{O}_{x}$ is a $K$-biorbitope. First consider the action Example 8.6 on hermitian matrices, for $\mathbb{K}=\mathbb{R}, \mathbb{C}, \mathbb{H}$. Up to scaling and translation there is exactly one biorbitope $\mathcal{O}_{x}$ of this type, namely for $x=\operatorname{diag}(1,0, \ldots, 0)$. The orbit $K x$ consists of all psd rank one matrices of (reduced) trace 1 . Its convex hull has the rank condition removed: $\mathcal{O}_{x}=\{y: y \succeq 0$, $\operatorname{tr}(y)=1\}$.

8.13. For the remaining actions Examples 8.3, 8.7 and 8.8 there exist two essentially different biorbitopes. When $\mathbb{K}=\mathbb{R}$, we have to exclude the case $m=n$ in Example 8.3 and the case $n$ even in Example 8.7. Indeed, these are the cases when the restricted root system is of type $D$ (see Theorem 6.7). Otherwise, the two biorbitopes are:

(a) $x=(1,0, \ldots, 0)$ and $\mathcal{O}_{x}=\left\{y:\|y\|_{*} \leq 1\right\}$, the unit ball in the nuclear norm; (b) $x=(1, \ldots, 1)$ and $\mathcal{O}_{x}=\left\{y:\|y\|_{1} \leq 1\right\}$, the unit ball in the operator norm.

In case (b) of the action Example 8.3, the $K$-orbit $K x$ is the Stiefel manifold $V_{n}\left(\mathbb{K}^{m}\right)$ of orthonormal $n$-frames in $\mathbb{K}^{m}$, at least for $n<m$. We therefore call these orbitopes the Stiefel orbitopes. For $m=n$ and $\mathbb{K} \neq \mathbb{R}$, we get tautological orbitopes: $\mathcal{O}_{x}$ is the convex hull of $S U(n)$ (case $\mathbb{K}=\mathbb{C}$ ), resp. of $S p(n)$ (case $\mathbb{K}=\mathbb{H}$ ).

Acknowledgements Supported by DFG Grants SCHE281/10- $n(n=1,2)$, partially supported by EU Horizon 2020 program, Grant Agreement 813211 (POEMA). All support is gratefully acknowledged. We are also grateful to an anonymous referee for valuable comments.

Funding Open Access funding enabled and organized by Projekt DEAL.

Open Access This article is licensed under a Creative Commons Attribution 4.0 International License, which permits use, sharing, adaptation, distribution and reproduction in any medium or format, as long as you give appropriate credit to the original author(s) and the source, provide a link to the Creative Commons licence, and indicate if changes were made. The images or other third party material in this article are included in the article's Creative Commons licence, unless indicated otherwise in a credit line to the material. If material is not included in the article's Creative Commons licence and your intended use is not permitted by statutory regulation or exceeds the permitted use, you will need to obtain permission directly from the copyright holder. To view a copy of this licence, visit http://creativecommons.org/ licenses/by/4.0/.

\section{References}

[1] Biliotti, L., Ghigi, A., Heinzner, P.: Polar orbitopes. Commun. Anal. Geom. 21, 579-606 (2013)

[2] Biliotti, L., Ghigi, A., Heinzner, P.: Coadjoint orbitopes. Osaka J. Math. 51, 935-968 (2014)

[3] Borel, A., Tits, J.: Groupes réductifs. Publ. Math. I. H. E. S. 27, 55-151 (1965)

[4] Bump, D.: Lie Groups, 2nd edn. Graduate Texts in Mathematics, vol. 225. Springer, New York (2013) 
[5] Casselman, W.A.: Geometric rationality of Satake compactifications. In: Algebraic Groups and Lie Groups, Australian Mathematical Society Lecture Series, vol. 9. Cambridge, pp. 81-103 (1997)

[6] Chua, C.B.: Relating homogeneous cones and positive definite cones via $T$-algebras. SIAM J. Optim. 14, 500-506 (2003)

[7] Dadok, J.: Polar coordinates induced by actions of compact Lie groups. Trans. Am. Math. Soc. 288, 125-137 (1985)

[8] Fan, K.: Maximum properties and inequalities for the eigenvalues of completely continuous operators. Proc. Nat. Acad. Sci. USA 37, 760-766 (1951)

[9] Farenick, D.R., Pidkowich, B.A.F.: The spectral theorem in quaternions. Linear Algebra Appl. 371, 75-102 (2003)

[10] Horn, R.A., Johnson, Ch.R.: Matrix Analysis. 2nd edn. Cambridge (2013)

[11] Knapp, A.W.: Lie Groups Beyond an Introduction, 2nd edn. Progress in Mathematics, vol. 140. Birkhäuser, Boston (2005)

[12] Kobert, T.: Spectrahedral and Semidefinite Representability of Orbitopes, Ph. D. thesis. University of Konstanz (2018)

[13] Kostant, B.: On convexity, the Weyl group and the Iwasawa decomposition. Ann. Sci. E. N. S. 4(6), 413-455 (1973)

[14] Leite, R.S., Richa, T.R.W., Tomei, C.: Geometric proofs of some theorems of SchurHorn type. Linear Algebra Appl. 286, 149-173 (1999)

[15] Lusztig, G., Tits, J.: The inverse of a Cartan matrix. Ann. Univ. Timis. 30, 17-23 (1992)

[16] Onishchik, A.L., Vinberg, E.B.: Lie Groups and Lie Algebras III. Structure of Lie Groups and Lie Algebras. Encyclopedia of Mathematical Sciences, vol. 41. Springer, Berlin (1994)

[17] Ortega, J.-P., Ratiu, T.S.: Momentum Maps and Hamiltonian Reduction. Progress in Mathematics, vol. 222. Birkhäuser, Boston (2004)

[18] Sanyal, R., Sottile, F., Sturmfels, B.: Orbitopes. Mathematika 57, 275-314 (2011)

[19] Satake, I.: On representations and compactifications of symmetric Riemannian symmetric spaces. Ann. Math. 71, 77-110 (1960)

[20] Saunderson, J., Parrilo, P.A., Willsky, A.S.: Semidefinite descriptions of the convex hull of rotation matrices. SIAM J. Optim. 25, 1314-1343 (2015)

[21] Scheiderer, C.: Spectrahedral shadows. SIAM J. Appl. Algebra Geom. 2, 26-44 (2018)

[22] Schneider, R.: Convex Bodies: The Brunn-Minkowski Theory, Second expanded edition. Cambridge (2014)

[23] Sinn, R.: Algebraic boundaries of $S O$ (2)-orbitopes. Discrete Comput. Geom. 50, 219 $235(2013)$

[24] Tits, J.: Tabellen zu den einfachen Lie Gruppen und ihren Darstellungen, Lecture Notes in Mathematics, vol. 40. Springer, Berlin (1967)

[25] Vinberg, E.B.: The theory of homogeneous convex cones. (English translation). Trans. Mosc. Math. Soc. 12, 340-403 (1965)

[26] Zhang, F.: Quaternions and matrices of quaternions. Linear Algebra Appl. 251, 21-57 (1997)

Publisher's Note Springer Nature remains neutral with regard to jurisdictional claims in published maps and institutional affiliations. 